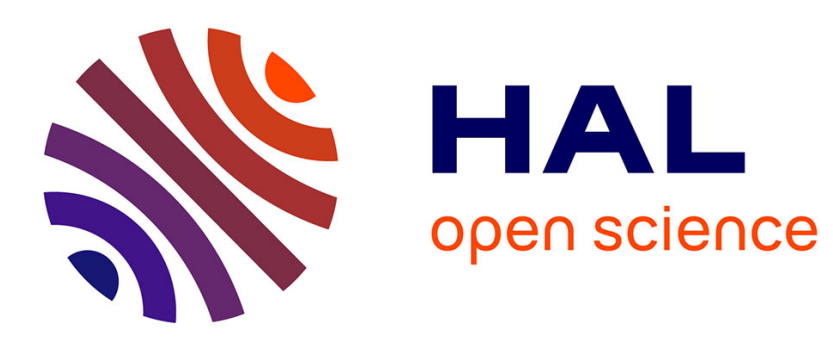

\title{
Condition-based maintenance with imperfect inspections for continuous degradation processes
}

\author{
Songhua Hao, Jun Yang, Christophe Bérenguer
}

\section{To cite this version:}

Songhua Hao, Jun Yang, Christophe Bérenguer. Condition-based maintenance with imperfect inspections for continuous degradation processes. Applied Mathematical Modelling, 2020, 86, pp.311-334. 10.1016/j.apm.2020.05.013 . hal-02860252

\section{HAL Id: hal-02860252 https://hal.science/hal-02860252}

Submitted on 9 Jun 2020

HAL is a multi-disciplinary open access archive for the deposit and dissemination of scientific research documents, whether they are published or not. The documents may come from teaching and research institutions in France or abroad, or from public or private research centers.
L'archive ouverte pluridisciplinaire HAL, est destinée au dépôt et à la diffusion de documents scientifiques de niveau recherche, publiés ou non, émanant des établissements d'enseignement et de recherche français ou étrangers, des laboratoires publics ou privés. 


\title{
Condition-based maintenance with imperfect inspections for continuous degradation processes
}

\author{
Songhua $\mathrm{Hao}^{1 *}$, Jun Yang ${ }^{2}$, Christophe Berenguer ${ }^{3}$ \\ ${ }^{1}$ School of Aeronautics and Astronautics, Sichuan University, Chengdu, China \\ ${ }^{2}$ School of Reliability and Systems Engineering, Beihang University, Beijing, China \\ ${ }^{3}$ Univ. Grenoble Alpes, CNRS, Grenoble INP, GIPSA-lab, Grenoble, France
}

\begin{abstract}
A condition-based maintenance (CBM) strategy is now recognized as an efficient approach to perform maintenance at the best time before failures so as to save lifetime cycle cost. For continuous degradation processes, a significant source of variability lies in measurement errors caused by imperfect inspections, and this may lead to "false positive" or "false negative" observations, and consequently to inopportune maintenance decisions. To the best of our knowledge, researches on $\mathrm{CBM}$ optimization with imperfect inspections remain limited for continuous degradation processes, even though the subject is of practical interest for the implementation of a CBM policy. Imperfect inspections are indeed imperfect but still return interesting information on the system degradation level, and making them perfect can be expensive. Therefore, we analyze the economic performance of a maintenance policy with imperfect inspections, and compare it with the classical policy with perfect inspections to see which policy offers the best benefit in a given situation. Furthermore, a CBM policy with a two-stage inspection scheme is proposed to take benefit of mixing both perfect and imperfect inspections in the same maintenance policy. Through numerical experiments and a real case study, it is shown that the policy with imperfect inspections can be better than the classical one, and that the proposed policy with a two-stage inspection scheme always leads to the minimum long run maintenance cost rate.
\end{abstract}

Key words: Continuous degradation process; condition-based maintenance; measurement error; imperfect inspection; two-stage inspection scheme; long run cost rate.

\section{Acronyms}

CBM condition-based maintenance

CDF cumulative distribution function

PDF probability density function

CM Corrective maintenance

PM Preventive maintenance

FP false positive

FN false negative

TP true positive

TN true negative

\footnotetext{
* Corresponding author.
} 


\section{Nomenclature}

$X(t) \quad$ Actual degradation level at time $t$

$\alpha \quad$ Shape parameter of the actual degradation process

$\Lambda(t) \quad$ Nonlinear drift function of the actual degradation process

$\beta \quad$ Rate parameter of the actual degradation process

$Z(t) \quad$ Inspected degradation level at time $t$

$\varepsilon(t) \quad$ Measurement error at time $t$

$\sigma_{\varepsilon} \quad$ Standard deviation of the measurement error

$f_{X}(x ; t) \quad$ PDF of $X(t)$

$\Gamma(u) \quad$ Gamma function with parameter $u$

$d_{C M} \quad$ Failure threshold level of the continuous degradation process

$d_{P M} \quad$ Failure threshold level for preventive maintenance

$S_{0} \quad$ Classical maintenance policy with perfect inspection scheme

$S_{A} \quad$ Maintenance policy with imperfect inspection scheme

$S_{B} \quad$ Maintenance policy with two-stage inspection scheme

$\tau \quad$ Periodic inspection interval of the maintenance policy

$M \quad$ Number of the last imperfect inspection of the maintenance policy $S_{B}$

$C_{P I} \quad$ Cost for each perfect inspection

$C_{I I} \quad$ Cost for each imperfect inspection

$C_{C M} \quad$ Cost for a corrective maintenance

$C_{P M} \quad$ Cost for a preventive maintenance

$C R \quad$ Long run cost rate of a maintenance policy

$E(C) \quad$ Expected cost of a renewal cycle

$E(L) \quad$ Expected length of a renewal cycle

$i \quad$ Number of the first inspection after the actual degradation path exceeds $d_{P M}$

$j \quad$ Number of the first inspection after the inspected degradation path exceeds $d_{P M}$

$T_{F} \quad$ System failure time without regard to maintenance activities

$k \quad$ Number of the first inspection after $T_{F}$

$F_{T}(t) \quad \mathrm{CDF}$ of the system failure time $T_{F}$

\section{Introduction}

Thanks to a deepening understanding of system failure mechanism, and rapid development of condition monitoring technology, degradation analysis has now become a main approach for 
reliability assessment and lifetime evaluation [1]. In fact, the failure of a system can often be attributed to some continuous degrading characteristics, e.g., material loss of a polymeric coating [2]. To well capture the dynamics in the underlying continuous degradation process, more and more researches have been focusing on stochastic degradation process models [3], including the Wiener process $[4,5]$, the gamma process $[6,7]$ and the inverse Gaussian process $[8,9]$.

From the practical viewpoint, degradation-based failures of a system may cause extra cost due to unscheduled system downtime, and also lead to uncontrolled hazards and damages to humans and environments. Confronted with this and thanks to the development of sensor technology, CBM has gained much popularity recently [10]. Compared to the classical time-based maintenance policy, $\mathrm{CBM}$ has been proven as an efficient strategy to perform maintenance at the right time before failure so as to save lifetime cycle cost [11]. Ahmad and Kamaruddin [12] surveyed the industrial applications of CBM strategy, and also explored some practical challenges in implementing it. Noortwijk [13] presented an extensive catalogue of inspection and maintenance models under gamma process degradation model. Alaswad and Xiang [14] reviewed the literature on CBM strategies based on stochastic degradation models.

Different from the CBM strategies for degradation processes with more explicit physical meaning, purely data-driven maintenance models have also gained much attention. For example, based on the collected data of pump speed and the junction chamber level, Zhang et.al [15] employed a neural network algorithm and a hierarchical particle swarm optimization algorithm to schedule the maintenance of pumps. Baptista et.al [16] integrated the ARMA model with datadriven techniques to predict fault events, and then make maintenance decisions for the aircraft engines. In fact, both degradation-based CBM models and data-driven maintenance models are aiming at predicting the risk of product failure in the future, and make maintenance decisions based on this. Therefore, these two techniques can be potentially combined into a hybrid maintenance model with data-driven performance prediction and degradation-based decision-making.

The selection of inspection schedule has obvious effect on the performance of a CBM strategy. Although continuous monitoring is the most effective method to preventively find system defects and trigger a warning [17], information on system states has to be provided in real time, and this will surely incur high inspection costs [18]. Compared to continuous monitoring, periodic inspections $[19,20]$ can be more cost effective, and more appropriate for some practical systems with limited measurement environment. Furthermore, non-periodic inspection scheme [21,22] has gained much attention in recent years, where upon each inspection, the next inspection interval is determined based on the current system state. In fact, non-periodic inspection scheme can lead to potential cost savings since the reschedule of inspection intervals as the system degrades, but it also needs more documentation work and is hard to implement in practice [14].

For continuous degradation processes, a significant source of variability lies in measurement errors caused by imperfect inspections [23], and many studies have been conducted on it. For example, Zhai et al. [24] considered inevitable measurement errors in degradation-based burn-in 
process, and optimized the cutoff levels for two burn-in models with different cost structures. To fulfill a specified lifetime estimation requirement for the Wiener degradation process, $\mathrm{Si}$ et al. formulated the permissible bias and standard deviation of the distribution-related measurement error [25]. By minimizing the test cost with a maximum acceptable approximate standard error, Zhang et al. optimized the amount of units and the measurement schedule for repeated degradation test planning considering measurement variability [26].

Within a CBM strategy, the decision to do nothing or to conduct preventive maintenance, is made according to the degradation performance inspection results. Most existing CBM strategies are based on the assumption of perfect inspections [27], which means that the inspections can perfectly return the true actual degradation level with no error. However, considering that measurement errors are inevitable in practice, an inspection can be imperfect in two ways [28]: a false positive (FP) occurs when the inspection reveals that maintenance is needed while in fact it is not, and a false negative (FN) occurs when the inspection reveals that maintenance is not needed while in fact it is. These two imperfect inspection results will lead to additional cost due to premature maintenance activity or unrevealed risk for future operation [29], and the performance of such a condition-based maintenance policy could be worse than that of a "blind" time-based preventive maintenance [30].

Recently, more and more studies have been conducted to investigate the CBM strategy with imperfect inspections, but mainly based on discrete degradation processes like a multi-state system [31]. For example, to detect both early and natural degradation-based failures, Berrade [32] developed a two-phase inspection policy with different frequencies, where false positives or false negatives may occur with given probabilities. For a system with three possible states: good, defective and failed, Berrade et al. [33] studied the imperfect inspection and replacement policy with constant probabilities of false positives or false negatives. Then Driessen et al. [34] extended this maintenance policy by considering time-varying probabilities of imperfect inspections. Besides imperfect inspections, Alberti et al. [35] also studied the impact of maintenance quality and the probability that an inspection may incur a defect. Levitin, Xing and Huang [36] proposed a probabilistic evaluation model for reliability metrics of a rescue operation mission, and also formulated the imperfect inspection schedule optimization problem with fixed mission time.

However, researches on CBM strategy with imperfect inspections for continuous degradation processes are limited. Liu et al. proposed an imperfect inspection policy for a multivariate Wiener degradation process, and any inspection may fail to discover a failure with given probability [37]. In the maintenance cost analysis framework in [38], false and missed alarm probabilities are defined within the concept of fault diagnosis and considered to be time-dependent, but have to be estimated with Mont Carlo simulation method. Huynh, Barros and Bérenguer presented an adaptive CBM decision framework under variable environment and imperfect inspections with measurement errors, and sensitivity analysis shows that the inevitable parameter estimation errors will clearly reduce the performance of the CBM strategy [39]. For a system subject to continuous degradation process with 
random measurement errors, Shen and Cui developed a new inspection scheme mixing both perfect and imperfect inspections [40]. A dynamic inspection and maintenance policy was proposed in [41], where condition monitoring quality and replacement decisions are jointly adjusted at each inspection.

From the viewpoint of availability and economy, a system is expected to be in continuous operation. Therefore, downtime for degradation model parameter updating and CBM strategy decision making may not be always practical, and this strategy will also cause reduction of CBM strategy performance due to estimation errors. Under this circumstance, this paper studies CBM policies with imperfect inspections for continuous degradation processes. First, a CBM maintenance policy based only on imperfect inspections is studied: decisions are made directly upon imperfect inspections, which are imperfect but still return interesting information on the system degradation levels. Therefore, it can be more beneficial to use imperfect inspections at a lower cost rather than perfect inspections at a higher cost. Another main contribution of this work is the mixture of both perfect and imperfect inspections in the same maintenance policy, so as to adapt the quality and the cost of the inspection to the actual need for decision-making purposes.

To the best of our knowledge, our work is one of the few that relax the assumption of perfect inspection, and consider explicitly the case of imperfect inspection in the maintenance model and its optimization: it is thus a first step towards a better on-field applicability of condition-based maintenance models. Furthermore, beyond the resulting model itself, we are convinced that one of the main managerial and application interests of this work lies on the proposed approach aiming at considering the possible imperfect inspections and also the mixture of both perfect and imperfection inspections in the CBM modelling and optimization. Such an approach also contributes to open research issues aiming at a better applicability of condition-based maintenance.

The rest of this article is in the following structure. Section 2 formulates the modelling assumptions on the continuous degradation process, the imperfect inspection scheme and the maintenance operations considered in this work. Two CBM strategies considering imperfect inspections are developed in Sections 3, respectively with a purely imperfect inspection scheme and a two-stage inspection scheme. Through numerical experiments and a real case study, Section 4 compares the proposed maintenance policies with the classical one with perfect inspections. Finally, some conclusions and perspectives on future researches are drawn in Section 5.

\section{Degradation and maintenance operations: modelling assumptions}

\subsection{Continuous degradation process modelling}

In this paper, we focus on systems subject to continuous degradation phenomena, whose degradation can be characterized by a single degradation index. The evolution of this index can be modelled by a stochastic process. Among the mentioned three popular stochastic degradation process models, the gamma process is the limit of a shot-noise process with exponential decay, and it is appropriate in describing monotonical degradation processes. The Wiener process is an almost 
surely continuous martingale, and it is suitable in modeling non-monotonic degradation processes. The inverse Gaussian process is a limiting compound Poisson Process, and it is flexible in incorporating random effects and covariates that account for heterogeneities, but has less applications than the gamma process. Specifically, the gamma degradation process model is studied in this manuscript for illustration, and other degradation models, like the Wiener process model and the inverse Gaussian process model, can be studied in a similar framework.

To design an optimal CBM strategy for the gamma degradation process, some basic formulations of the degradation model and maintenance policy are first presented as follows:

1) The system begins in a perfect state without any deterioration, i.e., $X(0)=0$;

2) The non-homogeneous gamma process is used to model the actual but hidden continuous degradation process $X(t)$, i.e., $X(t)-X(0) \sim G a(\alpha \Lambda(t), \beta)$, with the cumulative distribution function $(\mathrm{CDF})$ denoted by $F_{X}(x ; t)$ and the probability density function (PDF) is:

$$
f_{X}(x ; t)=\frac{\beta^{\alpha \Lambda(t)}}{\Gamma(\alpha \Lambda(t))} x^{\alpha \Lambda(t)-1} e^{-\beta x}
$$

where $\alpha$ is the shape parameter, $\Lambda(t)$ is the nonlinear drift function, $\beta$ is the rate parameter, and $\Gamma(u)=\int_{0}^{+\infty} x^{u-1} e^{-x} d x$ is the gamma function.

3) The system failure threshold level is denoted by $d_{C M}$, and a failure occurs as soon as the actual degradation process $X(t)$ first exceeds the failure threshold level $d_{C M}$.

\subsection{Maintenance modelling assumptions}

The development of the maintenance models proposed in this work is based on the following assumptions.

1) The system is periodically inspected with inspection interval $\tau$, i.e., at times $t_{m}=m \tau$; the inspection interval $\tau$ is used in the following as a decision variable to to be optimized to make the optimal inspection schedule.

2) Perfect inspections can be implemented on the system: they perfectly return the true actual degradation level with no error, and the cost for each perfect inspection is $C_{P I}$;

3) Imperfect inspections: the imperfectly inspected degradation level can be modelled by introducing an extra measurement error term, and the cost for each imperfect inspection is $C_{I I}$.

$$
Z\left(t_{m}\right)=X\left(t_{m}\right)+\varepsilon\left(t_{m}\right)
$$

where $t_{m}=m \tau$ is the $m^{t h}$ inspection time, $\tau$ is the inspection interval, $X_{m}=X\left(t_{m}\right)$, $Z_{m}=Z\left(t_{m}\right)$ and $\varepsilon_{m}=\varepsilon\left(t_{m}\right) \sim N\left(0, \sigma_{\varepsilon}^{2}\right)$ are respectively the actual degradation level, the inspected degradation level and the measurement error at time $t_{m}$.

4) Corrective maintenance $(\mathrm{CM})$ : the system failure is self-announcing, and can be found out 
as soon as it happens, then a corrective maintenance is immediately conducted. The cost for a corrective maintenance is $C_{C M}$;

5) Preventive maintenance (PM): after each inspection before the system fails, a preventive maintenance is carried out whenever the inspected degradation level is greater than $d_{P M}$. Otherwise, we do nothing and let the system continue working. A preventive maintenance incurs a cost $C_{P M}$. $d_{P M}$ is used in the following as a decision variable to be optimized to make the best preventive maintenance decision.

6) Both corrective maintenance and preventive maintenance restore the system to an as-goodas new state. Durations for inspection, corrective maintenance and preventive maintenance are rather short compared to the operation time, and are assumed to be negligible in this paper.

7) Cost for inspections and maintenance actions usually satisfy a practical constraint that $C_{I I}<C_{P I}<C_{P M}<C_{C M}$

8) Measurement error probabilities

For any imperfect inspection, according to both the actual degradation level and the inspected results, there are four possible results: true positive (TP), false positive (FP), true negative (TN), false negative (FN), as shown in Table 1, where "negative" means that the inspection indicates that the observed degradation level is no larger than the preventive maintenance threshold level $d_{P M}$, and "positive" means the opposite. A "true positive" inspection outcome indicates that the degradation level is inspected to be larger than the preventive maintenance threshold level when it actually is, where as a "false positive" outcome indicates that the degradation level is inspected to be larger than the preventive maintenance threshold level when it actually is not.

Table 1. Possible results for any imperfect inspection

\begin{tabular}{|c|c|c|c|}
\hline \multicolumn{2}{|c|}{ Inspection result } & \multicolumn{2}{c|}{ the actual degradation level } \\
\cline { 3 - 4 } & Positive & Negative \\
\hline the inspected & Positive & TP & FP \\
\cline { 2 - 4 } degradation level & Negative & FN & TN \\
\hline
\end{tabular}

Accordingly, the occurrence probabilities for all four possible results for the $m$ th imperfect inspection can be computed as follows:

$$
\begin{gathered}
P\left(T P_{m}\right)=P\left\{d_{P M}<X_{m} \leq d_{C M}, d_{P M}<Z_{m}=X_{m}+\varepsilon_{m} \leq d_{C M}\right\} \\
P\left(F P_{m}\right)=P\left\{X_{m} \leq d_{P M}, d_{P M}<Z_{m}=X_{m}+\varepsilon_{m} \leq d_{C M}\right\} \\
P\left(T N_{m}\right)=P\left\{X_{m} \leq d_{P M}, Z_{m}=X_{m}+\varepsilon_{m} \leq d_{P M}\right\} \\
P\left(F N_{m}\right)=P\left\{d_{P M}<X_{m} \leq d_{C M}, Z_{m}=X_{m}+\varepsilon_{m} \leq d_{P M}\right\}
\end{gathered}
$$

where $T P_{m}, F P_{m}, T N_{m}$ and $F N_{m}$ denote possible results for the $m^{\text {th }}$ imperfect inspection. 


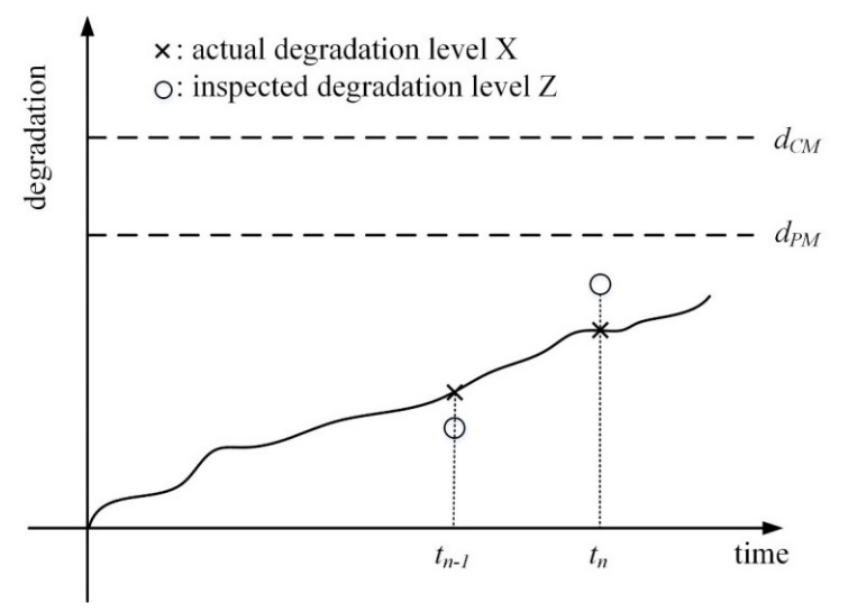

Figure 1. Schematic diagram for the conditional event $T N_{n} \mid T N_{n-1}$

Since the system degradation is modelled by the gamma process, degradation increments on disjointed time intervals are independent, but the cumulative degradation levels at different time points are not independent. Therefore, it is necessary to derive the probability of the conditional event for two successive inspection results, e.g., $T N_{n} \mid T N_{n-1}, n \geq 2$ shown in Figure 1:

$$
\begin{aligned}
P\left(T N_{n} \mid T N_{n-1}\right)= & P\left\{X_{n} \leq d_{P M}, Z_{n} \leq d_{P M} \mid X_{n-1} \leq d_{P M}, Z_{n-1} \leq d_{P M}\right\} \\
= & \frac{P\left\{X_{n} \leq d_{P M}, Z_{n} \leq d_{P M}, X_{n-1} \leq d_{P M}, Z_{n-1} \leq d_{P M}\right\}}{P\left\{X_{n-1} \leq d_{P M}, Z_{n-1} \leq d_{P M}\right\}} \\
& =\frac{\int_{0}^{d_{P M}} P\left\{x+\Delta X_{n-1, n} \leq d_{P M}, x+\Delta X_{n-1, n}+\varepsilon_{n} \leq d_{P M}, x+\varepsilon_{n-1} \leq d_{P M}\right\} f_{X_{n-1}}(x) d x}{\int_{0}^{d_{P M}} P\left\{x+\varepsilon_{n-1} \leq d_{P M}\right\} f_{X_{n-1}}(x) d x} \\
& =\frac{\int_{0}^{d_{P M}} \int_{0}^{d_{P M}-x} P\left\{y+\varepsilon_{n} \leq d_{P M}-x\right\} f_{\Delta X_{n-1, n}}(y) d y \cdot P\left\{\varepsilon_{n-1} \leq d_{P M}-x\right\} f_{X_{n-1}}(x) d x}{\int_{0}^{d_{P M}} \Phi\left(\frac{d_{P M}-x}{\sigma_{\varepsilon}}\right) f_{X_{n-1}}(x) d x} \\
& =\frac{\int_{0}^{d_{P M}} \int_{0}^{d_{P M}-x} \Phi\left(\frac{d_{P M}-x-y}{\sigma_{\varepsilon}}\right) f_{\Delta X_{n-1, n}}(y) d y \cdot \Phi\left(\frac{d_{P M}-x}{\sigma_{\varepsilon}}\right) f_{X_{n-1}}(x) d x}{\int_{0}^{d_{P M}} \Phi\left(\frac{d_{P M}-x}{\sigma_{\varepsilon}}\right) f_{X_{n-1}}(x) d x}
\end{aligned}
$$

where $\Delta X_{n-1, n}=X_{n}-X_{n-1} \sim G a\left(\alpha\left(\Lambda\left(t_{n}\right)-\Lambda\left(t_{n-1}\right)\right), \beta\right) \quad$ and $\quad X_{n-1} \sim G a\left(\alpha \Lambda\left(t_{n-1}\right), \beta\right)$ are both gamma distributed variables, whose PDFs are denoted by $f_{\Delta X_{n-1, n}}(y)$ and $f_{X_{n-1}}(x)$.

Furthermore, by changing the limits of integration or the integral functions, the occurrence probabilities of other conditional events for two successive inspection results can also be derived, and the results are presented in the Appendix.

\section{Two CBM strategies considering imperfect inspections}

\subsection{Description of the considered CBM strategies}

In the case of imperfect inspection, we propose to study two policies: the first one is classical 
periodic inspection/replacement policy, but with imperfect inspection: when compared to a classical periodic inspection/replacement policy with perfect inspections, it makes replacement decisions based on the imperfect information returned by imperfect inspections, at a lower cost. Such a "naive" policy can be interesting if the quality/cost ratio of the imperfect inspection is "favorable", which has to be determined based on mathematical cost analysis. This policy can be optimized using the two decision variables $d_{P M}$ and $\tau$.

The second maintenance policy is one of the main contributions of this work and it aims at taking benefit of the possibility to mix both perfect and imperfect inspections in the same maintenance policy so as to adapt the quality and the cost of the inspection to the actual need for decision-making purposes. As the system degrades over time, the degradation process is approaching the PM and CM threshold levels, which leads to increasing occurrence probabilities of FN and FP. Consequently, when the degradation level comes close to the PM and CM thresholds, it can be more beneficial to use more expensive, but also perfect inspections, to make more accurate and better informed maintenance decisions in order to avoid the cost induced by FN or FP. Based on this rationale, we propose a new two-stage inspection scheme, where the first $M$ inspections are imperfect, and the inspections from the $(M+1)^{\text {th }}$ one turns to be perfect. This policy can be optimized using the three decision variables $d_{P M}, \tau$ and $M$, which offers one additional degree of freedom when compared to the previous policy to find an optimal setting.

Denote the classical policy with perfect inspection scheme by $S_{0}$, the policy with imperfect inspection scheme by $S_{A}$, and the policy with two-stage inspection scheme by $S_{B}$. It can be indicated that the policy $S_{B}$ with $M=0$ is equivalent to the classical policy $S_{0}$, and the policy $S_{B}$ with large enough $M$ becomes equivalent to the proposed policy $S_{A}$ in Section 3. Therefore, it can be inferred that the optimally designed $S_{B}$ is worse than the optimally designed $S_{0}$ or $S_{A}$.

\subsection{Development of the maintenance costs models}

Maintenance cost is often used as a criterion to evaluate the performance and to optimize a CBM policy. Considering that each maintenance action (preventive or corrective replacement) restores the system to an as-good-as-new state, the maintained system deterioration process can be considered as a renewal process [42], and the long run cost rate over an infinite time horizon can be obtained through the renewal cycle theorem. In our setting, a renewal cycle can be defined as the interval between two replacements, either preventive or corrective. Therefore, the long run cost rate is defined as follows [43]:

$$
C R=\lim _{t \rightarrow \infty} \frac{C(t)}{t}=\frac{E(C)}{E(L)}
$$

where $E(C)$ and $E(L)$ are respectively the expected cost and length of a renewal cycle.

For the classical policy with perfect inspection scheme $S_{0}$, its expected cost and length of a 
renewal cycle are respectively as follows [44]:

$$
\begin{aligned}
& E\left(C_{0}\right)=C_{C M}+\left(C_{C M}-C_{P M}\right) M\left(d_{P M}\right) \\
&-\left(C_{C M}-C_{P M}-C_{I}\right)\left[F_{X}\left(d_{C M} ; \tau\right)+\int_{0}^{d_{P M}} m(x) F_{X}\left(d_{C M}-w ; \tau\right) d w\right] \\
& L\left(C_{0}\right)=\int_{0}^{\tau} F_{X}\left(d_{C M} ; t\right) d t+\int_{0}^{d_{P M}} m(x) \int_{0}^{\tau} F_{X}\left(d_{C M}-x ; t\right) d t d w
\end{aligned}
$$

where $F_{X}(x ; t)$ is the $\mathrm{CDF}$ of variable $X(t), M(x)=\sum_{i=1}^{+\infty} F_{X}(x ; i \cdot \tau)$ and $m(x)=M^{\prime}(x)$.

Based on $E\left(C_{0}\right)$ and $L\left(C_{0}\right)$, we can compute the long run cost rate $C R_{0}$ for the classical policy $S_{0}$ with any values of the inspection interval $\tau$ and preventive maintenance threshold level $d_{P M}$.

For the two proposed maintenance policies in this paper, a renewal cycle may also end up with either corrective or preventive maintenance. However, to calculate the probabilities of degradation paths in different cases of the renewal cycle, it is necessary to derive of the probability of the conditional event for two successive inspection results, as can be referred to the example of $T N_{n} \mid T N_{n-1}, n \geq 2$ in Equation (7).

In the following two subsections, we calculate the long run cost rate for the maintenance policy with purely imperfect inspection scheme and two-stage inspection scheme, respectively. Based on the cost analysis results, the optimal decision variables can be obtained.

\subsubsection{Cost analysis for the maintenance policy with imperfect inspection scheme}

For this maintenance policy with imperfect inspections, denoted by $S_{A}$, inspections are scheduled with fixed inspection interval $\tau$. According to different relationship between the sizes of inspected and actual degradation levels, the renewal cycle for policy $S_{A}$ may end up with either $\mathrm{CM}$ or PM. Therefore, by deriving the expected length and cost for the renewal cycle in different cases, we can obtain the long run cost rate for $S_{A}$, and then design the optimal inspection interval $\tau$ and preventive maintenance threshold level $d_{P M}$.

Let $i$ be the number of the first inspection after the actual degradation path exceeds $d_{P M}$, and $j$ be the number of the first inspection after the inspected degradation path exceeds $d_{C M}$. Besides, without regard to maintenance activities, system failure occurs at $T_{F}$, and $k=\left\lfloor T_{F} / \tau\right\rfloor+1$ is the number of the first inspection after $T_{F}$, i.e., $k$ is the number of the first inspection after the actual degradation path exceeds $d_{C M}$. Therefore, due to the fact that $0<d_{P M} \leq d_{C M}, i$ and $k$ have a primary constraint that $1 \leq i \leq k$. Furthermore, according to the relationship of size between $i, j$ and $k$, different cases for the renewal cycle are derived as follows.

Case A1: The cycle ends up with CM.

In this case, failure occurs before the $j^{\text {th }}$ inspection time when it is inspected to conduct PM, 
i.e., $j \geq k$. Considering that FNs may happen for the imperfect inspections before failures, this case can be further divided into two subcases:

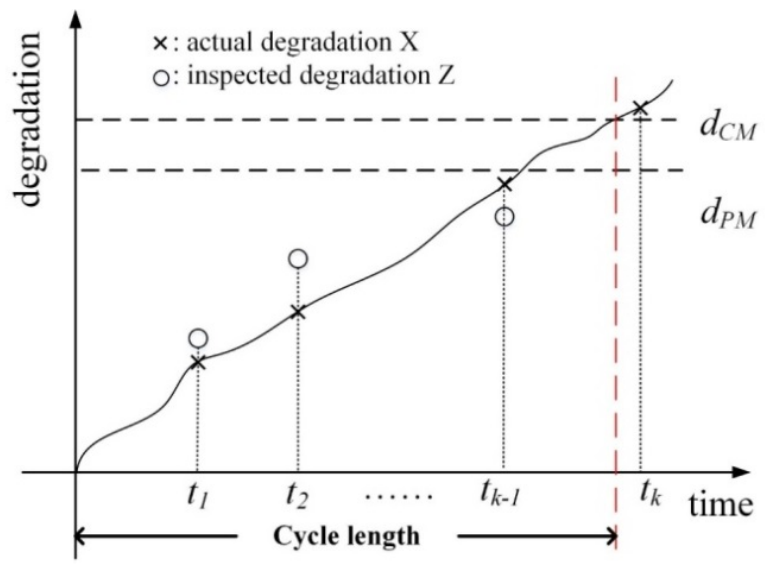

Figure 2. Schematic diagram for case A1.1

A1.1 $(j \geq k, i=k)$ : As shown in Figure 2, the actual degradation path exceeds both $d_{P M}$ and $d_{C M}$ in the same time interval $[(k-1) \tau, k \tau]$, and the inspected degradation does not exceed $d_{P M}$ by the $(k-1)^{t h}$ inspection.

Therefore, based on the probabilities of conditional events derived in Section 2, the occurrence probability of this subcase A1.1, on conditional of $T_{F} \in(0,+\infty)$, is as follows:

$$
\begin{aligned}
P_{A 1.1}\left(T_{F}\right) & =P\{j \geq k, i=k\} \\
& =P\left\{T N_{1}, T N_{2}, \cdots, T N_{k-1}, X_{k} \geq d_{C M}\right\} \\
& =P\left(T N_{1}\right) \cdot P\left(T N_{2} \mid T N_{1}\right) \cdots P\left(T N_{k-1} \mid T N_{k-2}\right) \cdot P\left(X_{k} \geq d_{C M} \mid T N_{k-1}\right)
\end{aligned}
$$

In addition, the expected cost and length for the renewal cycle in this subcase is:

$$
\begin{gathered}
E\left(C_{A 1.1} \mid T_{F}\right)=(k-1) C_{I I}+C_{C M} \\
E\left(L_{A 1.1} \mid T_{F}\right)=T_{F}
\end{gathered}
$$

A1.2 $(j \geq k, i<k)$ : As shown in Figure 3, the actual degradation path exceeds $d_{P M}$ in the time interval $[(i-1) \tau, i \tau]$, but this is not inspected to be truly positive by the $(k-1)^{t h}, k>i$ inspection. Besides, the actual degradation path exceeds $d_{C M}$ in the time interval $[(k-1) \tau, k \tau]$.

Note that in this subcase, the condition that $j \geq k, i<k$ and the primary constraint that $1 \leq i \leq k$ together leads to the constraint that $k>1$. 


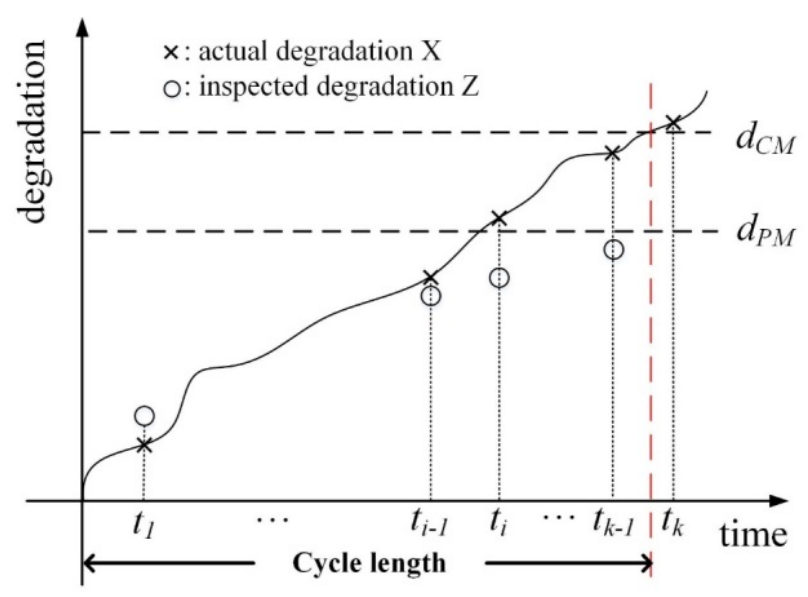

Figure 3. Schematic diagram for case A1.2

Therefore, based on the probabilities of conditional events derived in Section 2, the occurrence probability of this subcase A1.2, on conditional of $T_{F} \in(0,+\infty)$, is as follows:

$$
\begin{aligned}
P_{A 1.2}\left(T_{F}\right)= & P\{j \geq k, i<k\} \\
= & \sum_{i=1}^{k-1} P\left\{T N_{1}, \cdots, T N_{i-1}, F N_{i}, \cdots, F N_{k-1}, X_{k} \geq d_{C M}\right\} \\
= & \sum_{i=1}^{k-1}\left\{P\left(T N_{1}\right) \cdot P\left(T N_{2} \mid T N_{1}\right) \cdots P\left(T N_{i-1} \mid T N_{i-2}\right) \cdot P\left(F N_{i} \mid T N_{i-1}\right)\right. \\
& \left.\cdot P\left(F N_{i+1} \mid F N_{i}\right) \cdots P\left(F N_{k-1} \mid F N_{k-2}\right) \cdot P\left(X_{k} \geq d_{C M} \mid F N_{k-1}\right)\right\}
\end{aligned}
$$

In addition, the expected cost and length for the renewal cycle in this subcase is:

$$
\begin{gathered}
E\left(C_{A 1.2} \mid T_{F}\right)=(k-1) C_{I I}+C_{C M} \\
E\left(L_{A 1.2} \mid T_{F}\right)=T_{F}
\end{gathered}
$$

Case A2: The cycle ends up with PM.

In this case, failure occurs after the $j^{\text {th }}$ inspection time when it is inspected to conduct PM, i.e., $k>j$. Considering that FNs or FP may happen for the imperfect inspections, this case can be further divided into three subcases:

A2.1 $(k>j, i<j)$ : As shown in Figure 4, the actual degradation path exceeds $d_{P M}$ in the time interval $[(i-1) \tau, i \tau]$, but it is not inspected to be truly positive until the $j^{\text {th }}, j>i$ inspection. Also, the actual degradation path does not exceed $d_{C M}$ at the $j^{\text {th }}$ inspection.

Note that in this subcase, the condition that $k>j, i<j$ and the primary constraint that $1 \leq i \leq k$ together leads to the constraint that $j>1$. 


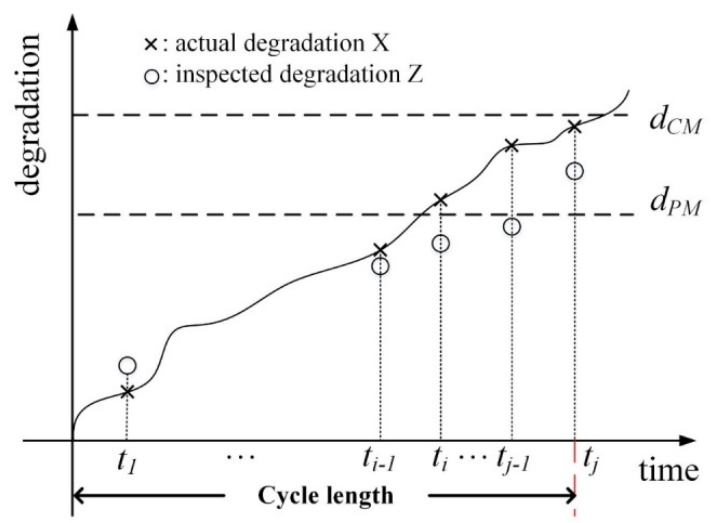

Figure 4. Schematic diagram for case A2.1

Therefore, based on the probabilities of conditional events derived in Section 2, the occurrence probability of this subcase A2.1, on conditional of $j=1,2, \cdots,+\infty$, is as follows:

$$
\begin{aligned}
P_{A 2.1}(j) & =P\{k>j, i<j\} \\
= & \sum_{i=1}^{j-1} P\left\{T N_{1}, \cdots T N_{i-1}, F N_{i}, \cdots, F N_{j-1}, T P_{j}\right\} \\
= & \sum_{i=1}^{j-1}\left\{P\left(T N_{1}\right) \cdot P\left(T N_{2} \mid T N_{1}\right) \cdots P\left(T N_{i-1} \mid T N_{i-2}\right)\right. \\
& \left.\cdot P\left(F N_{i} \mid T N_{i-1}\right) \cdots P\left(F N_{j-1} \mid F N_{j-2}\right) P\left(T P_{j} \mid F N_{j-1}\right)\right\}
\end{aligned}
$$

In addition, the expected cost and length for the renewal cycle in this subcase is:

$$
\begin{gathered}
E\left(C_{A 2.1} \mid j\right)=j C_{I I}+C_{P M} \\
E\left(L_{A 2.1} \mid j\right)=j \tau
\end{gathered}
$$

A2.2 $(k>j, i=j)$ : As shown in Figure 5, the actual degradation path exceeds $d_{P M}$ in the time interval $[(i-1) \tau, i \tau]$, and it is inspected to be truly positive at the same inspection time, i.e., $j=i$. Also, the actual degradation path does not exceed $d_{C M}$ at the $j^{\text {th }}$ inspection.

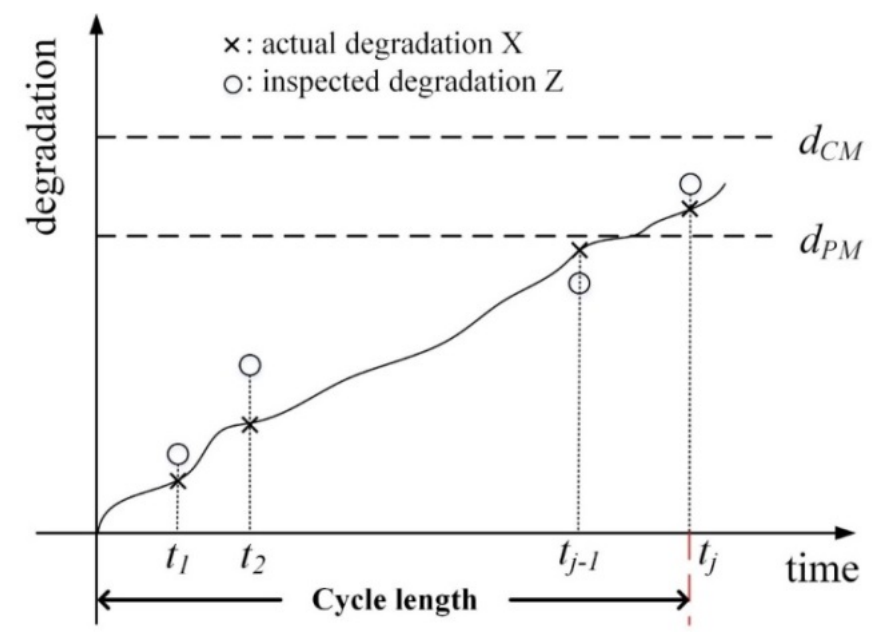

Figure 5. Schematic diagram for case A2.2

Therefore, based on the probabilities of conditional events derived in Section 2, the occurrence probability of this subcase A2.2, on conditional of $j=1,2, \cdots,+\infty$, is as follows: 


$$
\begin{aligned}
P_{A 2.2}(j) & =P\{k>j, i=j\} \\
& =P\left\{T N_{1}, \cdots T N_{j-1}, T P_{j}\right\} \\
& =P\left(T N_{1}\right) \cdot P\left(T N_{2} \mid T N_{1}\right) \cdots P\left(T N_{i-1} \mid T N_{i-2}\right) \cdot P\left(T P_{j} \mid T N_{j-1}\right)
\end{aligned}
$$

In addition, the expected cost and length for the renewal cycle in this subcase is:

$$
\begin{gathered}
E\left(C_{A 2.2} \mid j\right)=j C_{I I}+C_{P M} \\
E\left(L_{A 2.2} \mid j\right)=j \tau
\end{gathered}
$$

A2.3 $(k>j, i>j)$ : As shown in Figure 6, the actual degradation path does not exceed $d_{P M}$ by the $j^{\text {th }}$ inspection, but it is inspected to be falsely positive at $j \tau$.

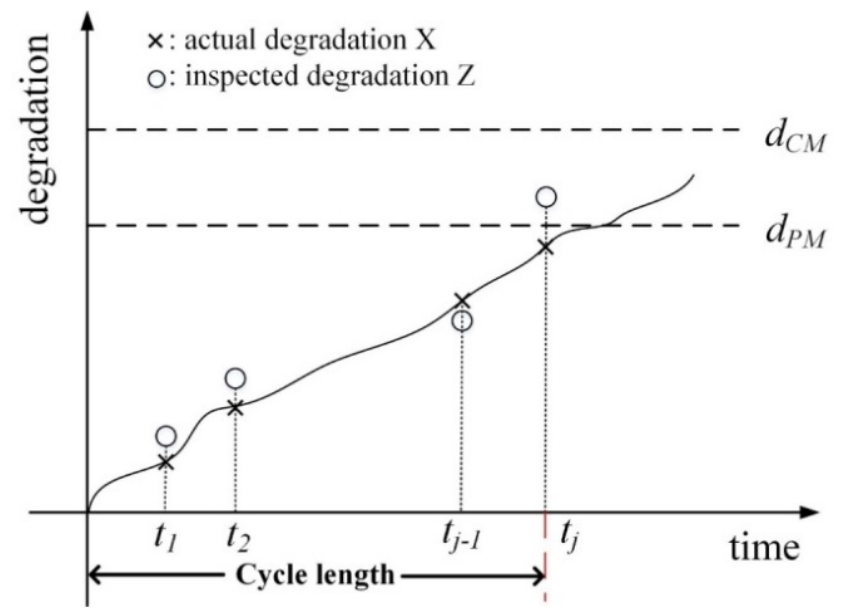

Figure 6. Schematic diagram for case A2.3

Therefore, based on the probabilities of conditional events derived in Section 2, the occurrence probability of this subcase $\mathrm{A} 2.3$, on conditional of $j=1,2, \cdots,+\infty$, is as follows:

$$
\begin{aligned}
P_{A 3.3}(j) & =P\{k>j, i>j\} \\
& =P\left\{T N_{1}, \cdots T N_{j-1}, F P_{j}\right\} \\
& =P\left(T N_{1}\right) \cdot P\left(T N_{2} \mid T N_{1}\right) \cdots P\left(T N_{i-1} \mid T N_{i-2}\right) \cdot P\left(F P_{j} \mid T N_{j-1}\right)
\end{aligned}
$$

In addition, the expected cost and length for the renewal cycle in this subcase is:

$$
\begin{gathered}
E\left(C_{A 2.3} \mid j\right)=j C_{I I}+C_{P M} \\
E\left(L_{A 2.3} \mid j\right)=j \tau
\end{gathered}
$$

\section{Total maintenance cost of policy $S_{A}$ :}

To account for the total long run cost rate for the proposed policy $S_{A}$, first we have to verify that the above cases and subcases are mutually exclusive events. In fact, we believe that this condition holds because $i$ is the number of the first inspection after the actual degradation path exceeds $d_{P M}$, $j$ is the number of the first inspection after the inspected degradation path exceeds $d_{C M}$, and $k$ is the number of the first inspection after the actual degradation path exceeds $d_{C M}$. Considering that the above subcases involves different relationships of size between variables $i, j, k$, and that there is no repetitive 
situation in any two subcases, subcases A1.1, A.1.2, A2.1, A.2.2, and A.2.3 are believed to be mutually exclusive events.

Furthermore, the above subcases compose the whole events of maintenance policy $S_{A}$ becasuse that the sum of the occurrence probabilities of cases $\mathrm{A} 1$ and $\mathrm{A} 2$ is:

$$
\begin{aligned}
& P_{A 1}+P_{A 2} \\
& =P_{A 1.1}+P_{A 1.2}+P_{A 2.1}+P_{A 2.2}+P_{A 2.3} \\
& =P\{j \geq k, i=k\}+P\{j \geq k, i<k\}+P\{k>j, i<j\}+P\{k>j, i=j\}+P\{k>j, i>j\} \\
& =P\{j \geq k\}-P\{j \geq k, i>k\}+P\{k>j\} \\
& =1-P\{j \geq k, i>k\} \\
& =1
\end{aligned}
$$

where $P\{j \geq k, i>k\}=0$ because that the fact $0<d_{P M} \leq d_{C M}$ leads to a primary constraint that $1 \leq i=\left\lfloor d_{P M} / \tau\right\rfloor+1 \leq j=\left\lfloor d_{C M} / \tau\right\rfloor+1$, that is to say the case $i>k$ won't occur and $P(i>k)=0$.

In summary, by considering the distribution characteristic of system failure time $T_{F}$ and all possible values of the inspection number $j$ to conduct PM, and summing over all the above cases and subcases, we can obtain the expected cost and length for $S_{A}$ as Equations (24) and (25). Therefore, its long run cost rate $C R_{A}$ for policy $S_{A}$ can be formulated by the ratio of $E\left(C_{A}\right)$ and $E\left(L_{A}\right)$, and the optimal $\tau$ and $d_{P M}$ for $S_{A}$ can be designed through minimizing $C R_{A}$.

$$
\begin{aligned}
E\left(C_{A}\right)= & \int_{0}^{+\infty} E\left(C_{A 1} \mid T_{F}\right) \cdot P_{A 1}\left(T_{F}\right) d\left[F_{T}(t)\right]+\sum_{j=1}^{+\infty} E\left(C_{A 2} \mid j\right) \cdot P_{A 2}(j) \\
& =\int_{0}^{+\infty}\left[E\left(C_{A 1.1} \mid T_{F}\right) \cdot P_{A 1.1}\left(T_{F}\right)+E\left(C_{A 1.2} \mid T_{F}\right) \cdot P_{A 1.2}\left(T_{F}\right)\right] d\left[F_{T}(t)\right] \\
& +\sum_{j=1}^{+\infty}\left[E\left(C_{A 2.1} \mid j\right) \cdot P_{A 2.1}(j)+E\left(C_{A 2.2} \mid j\right) \cdot P_{A 2.2}(j)+E\left(C_{A 2.3} \mid j\right) \cdot P_{A 2.3}(j)\right] \\
E\left(L_{A}\right)= & \int_{0}^{+\infty} E\left(L_{A 1} \mid T_{F}\right) \cdot P_{A 1}\left(T_{F}\right) d\left[F_{T}(t)\right]+\sum_{j=1}^{+\infty} E\left(L_{A 2} \mid j\right) \cdot P_{A 2}(j) \\
& =\int_{0}^{+\infty}\left[E\left(L_{A 1.1} \mid T_{F}\right) \cdot P_{A 1.1}\left(T_{F}\right)+E\left(L_{A 1.2} \mid T_{F}\right) \cdot P_{A 1.2}\left(T_{F}\right)\right] d\left[F_{T}(t)\right] \\
& +\sum_{j=1}^{+\infty}\left[E\left(L_{A 2.1} \mid j\right) \cdot P_{A 2.1}(j)+E\left(L_{A 2.2} \mid j\right) \cdot P_{A 2.2}(j)+E\left(L_{A 2.3} \mid j\right) \cdot P_{A 2.3}(j)\right]
\end{aligned}
$$

where $F_{T}(t)$ is the CDF of the system failure time $T_{F}$ :

$$
F_{F}(t)=P\left\{T_{F}<t\right\}=P\left\{X(t)>d_{C M}\right\}=F_{X}\left(d_{C M} ; t\right)
$$

3.2.2 Cost analysis for the maintenance policy with two-stage inspection scheme

Similar to $S_{A}$, policy $S_{B}$ is also with fixed inspection interval $\tau$. Besides, its renewal cycle may also end up with either CM or PM, and the cycle termination can lie in either the perfectinspection stage or the imperfect-inspection stage. Therefore, we derive the occurrence probability, the expected length and cost for the renewal cycle of $S_{B}$ in the following cases. Denotations $i, j, k, T_{F}$ are the same with those in subsection 3.2.1. The optimization variables for policy $S_{B}$ 
include inspection interval $\tau$, preventive maintenance threshold level $d_{P M}$ and number of inspections $M$ for stage transition.

Case B1: The cycle ends up with CM.

In this case, failure occurs before the $j^{\text {th }}$ inspection time when it is inspected to conduct PM, i.e., $\quad j \geq k$. Considering that FNs may happen for the imperfect inspections before failure, and that the last inspection of the cycle may be either imperfect or perfect, this case can be further divided into three subcases:

B1.1 $(k \leq M+1, j \geq k, i=k)$ : As shown in Figure 7, the actual degradation path exceeds both $d_{P M}$ and $d_{C M}$ in the same time interval $[(k-1) \tau, k \tau]$ in the imperfect-inspection stage, i.e., $k \leq M+1$, and the inspected degradation does not exceed $d_{P M}$ by the $(k-1)^{t h}$ inspection.

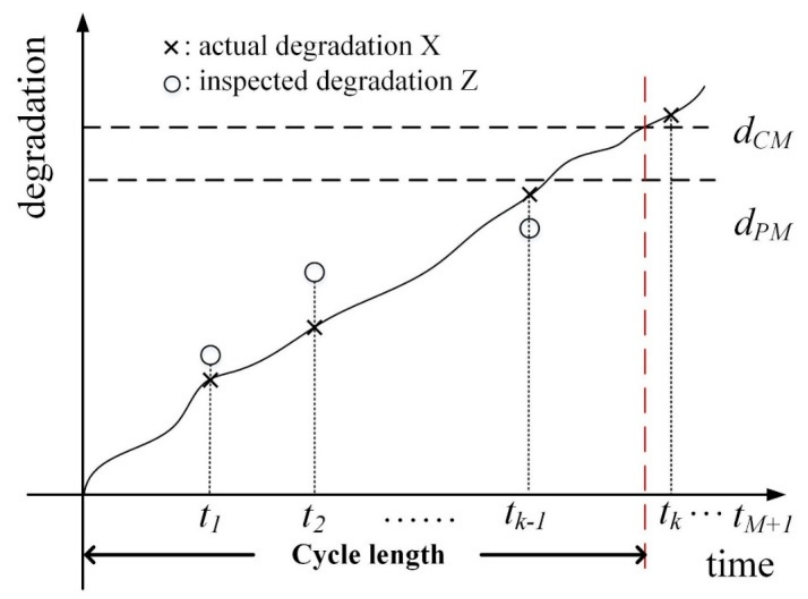

Figure 7. Schematic diagram for case B1.1

Therefore, based on the probabilities of conditional events derived in Section 2, the occurrence probability of this subcase $\mathrm{B} 1.1$, on conditional of $T_{S F} \in(0, M \tau)$, is as follows:

$$
\begin{aligned}
P_{B 1.1}\left(T_{F}\right) & =P\{k \leq M+1, j \geq k, i=k\} \\
& =P\left\{T N_{1}, T N_{2}, \cdots, T N_{k-1}, X_{k} \geq d_{C M}\right\} \\
& =P\left(T N_{1}\right) \cdot P\left(T N_{2} \mid T N_{1}\right) \cdots P\left(T N_{k-1} \mid T N_{k-2}\right) \cdot P\left(X_{k} \geq d_{C M} \mid T N_{k-1}\right)
\end{aligned}
$$

In addition, the expected cost and length for the renewal cycle in this subcase is:

$$
\begin{gathered}
E\left(C_{B 1.1} \mid T_{F}\right)=(k-1) C_{I I}+C_{C M} \\
E\left(L_{B 1.1} \mid T_{F}\right)=T_{F}
\end{gathered}
$$

B1.2 $(k \leq M+1, j \geq k, i<k)$ : As shown in Figure 8 , the actual degradation path exceeds $d_{P M}$ in the imperfect inspection interval $[(i-1) \tau, i \tau], i<M-1$, but this is not inspected to be truly positive by the $(k-1)^{t h}, i<k \leq M+1$ inspection. Besides, the actual degradation path exceeds $d_{C M}$ in the time interval $[(k-1) \tau, k \tau]$.

Note that in this subcase, the condition that $j \geq k, i<k$ and the primary constraint that 
$1 \leq i \leq k$ together leads to the constraint that $k>1$.

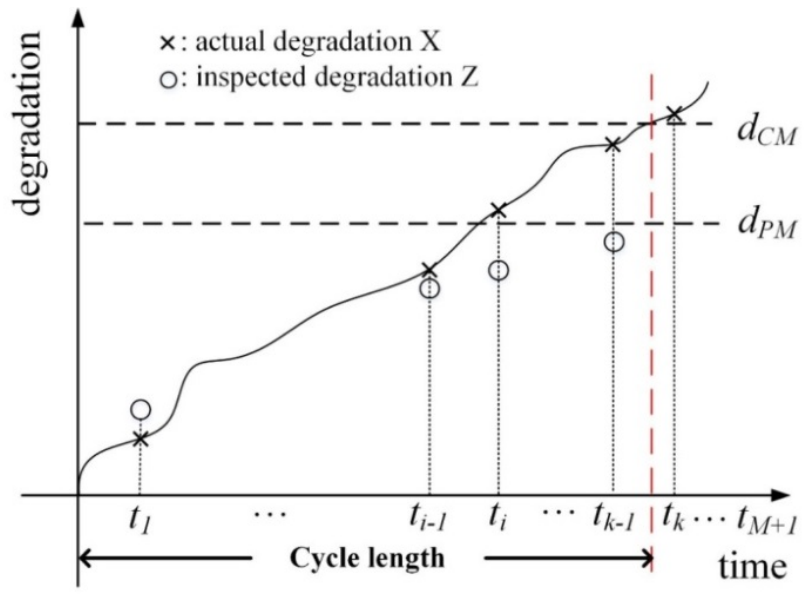

Figure 8. Schematic diagram for case B1.2

Therefore, based on the probabilities of conditional events derived in Section 2, the occurrence probability of this subcase $\mathrm{B} 1.2$, on conditional of $T_{F} \in(0, M \tau)$, is as follows:

$$
\begin{aligned}
P_{B 1.2}\left(T_{F}\right)= & P\{k \leq M+1, j \geq k, i<k\} \\
= & \sum_{i=1}^{k-1} P\left\{T N_{1}, \cdots, T N_{i-1}, F N_{i}, \cdots, F N_{k-1}, X_{k} \geq d_{C M}\right\} \\
= & \sum_{i=1}^{k-1}\left\{P\left(T N_{1}\right) \cdot P\left(T N_{2} \mid T N_{1}\right) \cdots P\left(T N_{i-1} \mid T N_{i-2}\right) \cdot P\left(F N_{i} \mid T N_{i-1}\right)\right. \\
& \left.\cdot P\left(F N_{i+1} \mid F N_{i}\right) \cdots P\left(F N_{k-1} \mid F N_{k-2}\right) \cdot P\left(X_{k} \geq d_{C M} \mid F N_{k-1}\right)\right\}
\end{aligned}
$$

In addition, the expected cost and length for the renewal cycle in this subcase is:

$$
\begin{gathered}
E\left(C_{B 1.2} \mid T_{F}\right)=(k-1) C_{I I}+C_{C M} \\
E\left(L_{B 1.2} \mid T_{F}\right)=T_{F}
\end{gathered}
$$

B1.3 $(k>M+1, i=j=k)$ : As shown in Figure 9, the actual degradation path exceeds $d_{C M}$ in the perfect inspection interval $[(k-1) \tau, k \tau], k>M+1$, which leads to the fact that the actual and perfectly inspected degradation path exceeds $d_{P M}$ in the same inspection interval, i.e., $i=j=k$, because if $i<k$ or $j<k$, then PM will be conducted before the system fails.

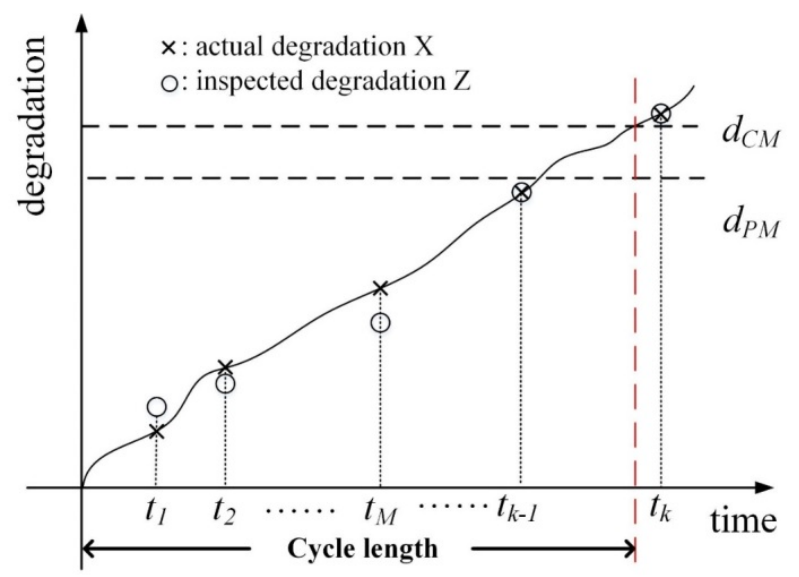

Figure 9. Schematic diagram for case B1.3 
Therefore, based on the probabilities of conditional events derived in Section 2, the occurrence probability of this subcase $\mathrm{B} 1.3$, on conditional of $T_{F} \in(0, M \tau)$, is as follows:

$$
\begin{aligned}
P_{B 1.3} & =P\{k>M+1, i=j=k\} \\
& =P\left\{T N_{1}, \cdots, T N_{M}, X_{k-1}<d_{P M}, X_{k} \geq d_{C M}\right\} \\
& =P\left(T N_{1}\right) \cdot P\left(T N_{2} \mid T N_{1}\right) \cdots P\left(T N_{M} \mid T N_{M-1}\right) \\
& \cdot P\left(X_{k-1}<d_{P M} \mid T N_{M}\right) \cdot P\left(X_{k} \geq d_{C M} \mid X_{k-1}<d_{P M}\right)
\end{aligned}
$$

In addition, the expected cost and length for the renewal cycle in this subcase is:

$$
\begin{gathered}
E\left(C_{B 1.3} \mid T_{F}\right)=M C_{I I}+(k-1-M) C_{P I}+C_{C M} \\
E\left(L_{B 1.3} \mid T_{F}\right)=T_{F}
\end{gathered}
$$

Case B2: The cycle ends up with inspected PM.

In this case, failure occurs after the $j^{\text {th }}$ inspection time when it is inspected to conduct PM, i.e., $k>j$. Considering that FNs or FP may happen for the imperfect inspections, and that the last inspection of the cycle may be either imperfect or perfect, this case can be divided into four subcases:

B2.1 $(j \leq M, k>j, i<j)$ : As shown in Figure 10, the actual degradation path exceeds $d_{P M}$ in the imperfect inspection interval $[(i-1) \tau, i \tau], i<M$, but it is not inspected to be truly positive until the $j^{\text {th }}, i<j \leq M$ imperfect inspection. Also, the actual degradation path does not exceed $d_{C M}$ at the $j^{\text {th }}$ inspection.

Note that in this subcase, the condition that $k>j, i<j$ and the primary constraint that $1 \leq i \leq k$ together leads to the constraint that $j>1$.

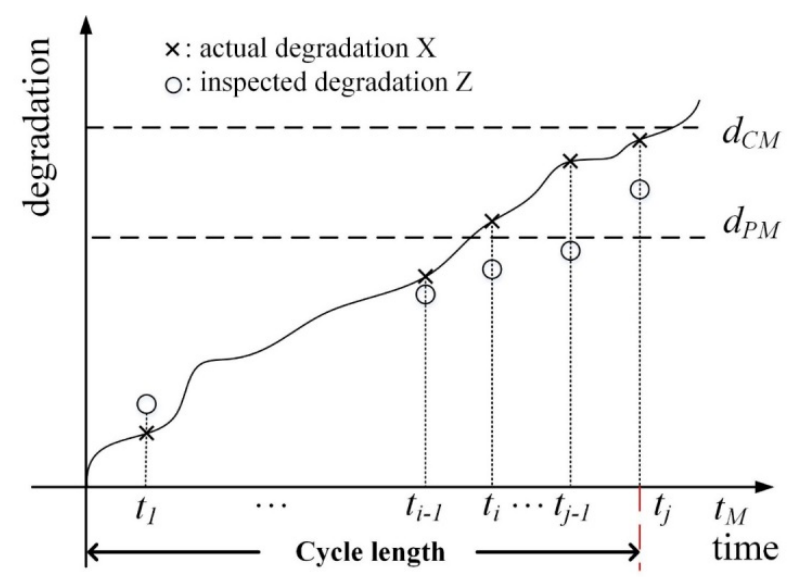

Figure 10. Schematic diagram for case B2.1

Therefore, based on the probabilities of conditional events derived in Section 2, the occurrence probability of this subcase B2.1, on conditional of $j=1,2, \cdots, M$, is as follows: 


$$
\begin{aligned}
P_{B 2.1}(j)= & P\{j \leq M, k>j, i<j\} \\
= & \sum_{i=1}^{j-1} P\left\{T N_{1}, \cdots T N_{i-1}, F N_{i}, \cdots, F N_{j-1}, T P_{j}\right\} \\
= & \sum_{i=1}^{j-1}\left\{P\left(T N_{1}\right) \cdot P\left(T N_{2} \mid T N_{1}\right) \cdots P\left(T N_{i-1} \mid T N_{i-2}\right)\right. \\
& \left.\cdot P\left(F N_{i} \mid T N_{i-1}\right) \cdots P\left(F N_{j-1} \mid F N_{j-2}\right) P\left(T P_{j} \mid F N_{j-1}\right)\right\}
\end{aligned}
$$

In addition, the expected cost and length for the renewal cycle in this subcase is:

$$
\begin{gathered}
E\left(C_{B 2.1} \mid j\right)=j C_{I I}+C_{P M} \\
E\left(L_{B 2.1} \mid j\right)=j \tau
\end{gathered}
$$

B2.2 $(j \leq M, k>j, i=j)$ : As shown in Figure 11, the actual degradation path exceeds $d_{P M}$ in the imperfect inspection interval $[(i-1) \tau, i \tau], i \leq M$, and it is inspected to be truly positive at the same time, i.e., $j=i$, and the actual degradation path does not exceed $d_{C M}$ at the $j \tau$.

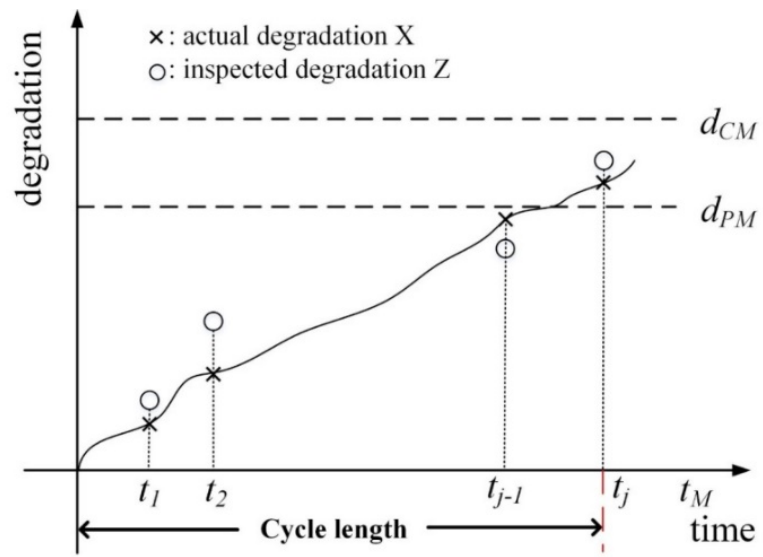

Figure 11. Schematic diagram for case B2.2

Therefore, based on the probabilities of conditional events derived in Section 2, the occurrence probability of this subcase $\mathrm{B} 2.2$, on conditional of $j=1,2, \cdots, M$, is as follows:

$$
\begin{aligned}
P_{B 2.2}(j) & =P\{j \leq M, k>j, i=j\} \\
& =P\left\{T N_{1}, \cdots T N_{j-1}, T P_{j}\right\} \\
& =P\left(T N_{1}\right) \cdot P\left(T N_{2} \mid T N_{1}\right) \cdots P\left(T N_{i-1} \mid T N_{i-2}\right) \cdot P\left(T P_{j} \mid T N_{j-1}\right)
\end{aligned}
$$

In addition, the expected cost and length for the renewal cycle in this subcase is:

$$
\begin{gathered}
E\left(C_{A 2.2} \mid j\right)=j C_{I I}+C_{P M} \\
E\left(L_{A 2.2} \mid j\right)=j \tau
\end{gathered}
$$

B2.3 $(j \leq M, k>j, i>j)$ : As shown in Figure 12, the actual degradation path does not exceed $d_{P M}$ by the $j^{\text {th }}$ imperfect inspection, but it is inspected to be falsely positive at $j \tau, j \leq M$. 


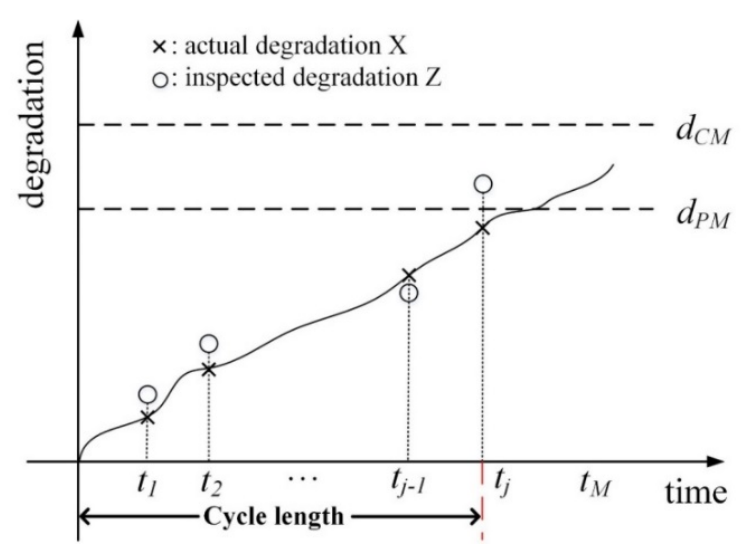

Figure 12. Schematic diagram for case $B 2.3$

Therefore, based on the probabilities of conditional events derived in Section 2, the occurrence probability of this subcase $\mathrm{B} 2.3$, on conditional of $j=1,2, \cdots, M$, is as follows:

$$
\begin{aligned}
P_{B 2.3}(j) & =P\{j \leq M, k>j, i>j\} \\
& =P\left\{T N_{1}, \cdots T N_{j-1}, F P_{j}\right\} \\
& =P\left(T N_{1}\right) \cdot P\left(T N_{2} \mid T N_{1}\right) \cdots P\left(T N_{i-1} \mid T N_{i-2}\right) \cdot P\left(F P_{j} \mid T N_{j-1}\right)
\end{aligned}
$$

In addition, the expected cost and length for the renewal cycle in this subcase is:

$$
\begin{gathered}
E\left(C_{A 2.3} \mid j\right)=j C_{I I}+C_{P M} \\
E\left(L_{A 2.3} \mid j\right)=j \tau
\end{gathered}
$$

B2.4 $(j \geq M+1, k>j, i=j)$ : As shown in Figure 13, the actual degradation path exceeds $d_{P M}$ in the perfect inspection interval $[(i-1) \tau, i \tau], i \geq M+1$, and it is inspected to be truly positive at the same time. Also, the actual degradation path does not exceed $d_{C M}$ at the $j^{\text {th }}$ inspection.

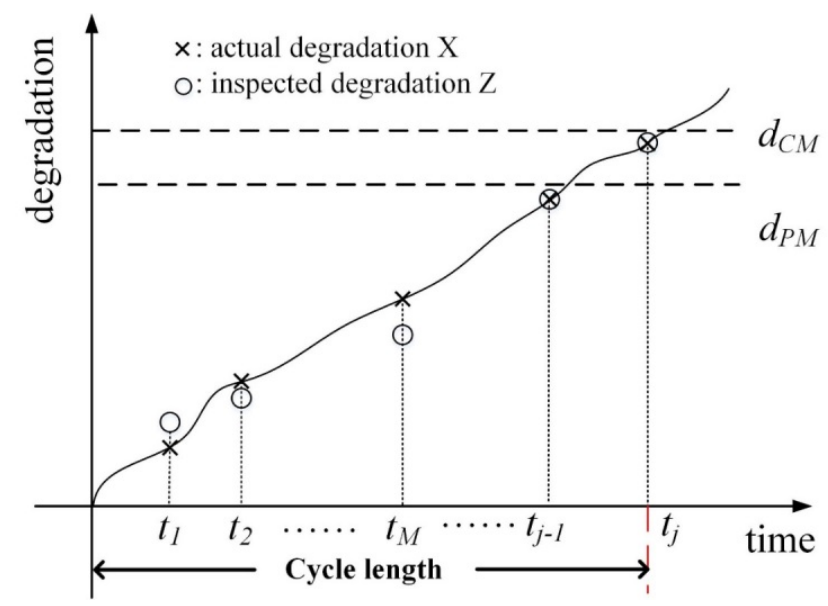

Figure 13. Schematic diagram for case B2.4

Therefore, based on the probabilities of conditional events derived in Section 2, the occurrence probability of this subcase $\mathrm{B} 2.4$, on conditional of $j=M+1, M+2, \cdots,+\infty$, is as follows: 


$$
\begin{aligned}
P_{B 2.4} & =P\{j \geq M+1, k>j, i=j\} \\
& =P\left\{T N_{1}, \cdots T N_{M}, X_{j-1}<d_{P M}, d_{P M} \leq X_{j}<d_{C M}\right\} \\
& =P\left(T N_{1}\right) \cdot P\left(T N_{2} \mid T N_{1}\right) \cdots P\left(T N_{M} \mid T N_{M-1}\right) \\
& \cdot P\left(X_{j-1}<d_{P M} \mid T N_{M}\right) \cdot P\left(d_{P M} \leq X_{j}<d_{C M} \mid X_{j-1}<d_{P M}\right)
\end{aligned}
$$

In addition, the expected cost and length for the renewal cycle in this subcase is:

$$
\begin{gathered}
E\left(C_{A 2.4} \mid j\right)=M C_{I I}+(j-M) C_{P I}+C_{P M} \\
E\left(L_{A 2.4} \mid j\right)=j \tau
\end{gathered}
$$

Total maintenance cost of policy $S_{B}$ :

To account for the total long run cost rate for the proposed policy $S_{B}$, first we have to verify that the above cases and subcases are mutually exclusive events. Similar to the situation of policy $S_{B}$, the subcases B1.1, B.1.2, B.1.3, B2.1, B.2.2, B.2.3 and B.2.4 involves different relationships of size between variables $i, j, k, M$, and that there is no repetitive situation in any two subcases. Therefore, these subcases are believed to be mutually exclusive events.

Furthermore, the above subcases compose the whole events of maintenance policy $S_{B}$ becasuse that the sum of the occurrence probabilities of cases $\mathrm{B} 1$ and $\mathrm{B} 2$ is:

$$
\begin{aligned}
& P_{B 1}+P_{B 2} \\
& =P_{B 1.1}+P_{B 1.2}+P_{B 1.3}+P_{B 2.1}+P_{B 2.2}+P_{B 2.3}+P_{B 2.4} \\
& =P\{k \leq M+1, j \geq k, i=k\}+P\{k \leq M+1, j \geq k, i<k\}+P\{k>M+1, i=j=k\} \\
& +P\{j \leq M, k>j, i<j\}+P\{j \leq M, k>j, i=j\}+P\{j \leq M, k>j, i>j\}+P\{j \geq M+1, k>j, i=j\} \\
& =P\{k \leq M+1, j \geq k\}-P\{k \leq M+1, j \geq k, i>k\}+P\{k>M+1, j \geq k\}-P\{k>M+1, j>k\} \\
& -P\{k>M+1, j=k, i \neq k\}+P\{j \leq M, k>j\}+P\{j \geq M+1, k>j\}-P\{j \geq M+1, k>j, i \neq j\} \\
& =P\{j \geq k\}-[P\{k \leq M+1, j \geq k, i>k\}+P\{k>M+1, j>k\}+P\{k>M+1, j=k, i \neq k\}] \\
& +P\{k>j\}-P\{j \geq M+1, k>j, i \neq j\} \\
& =1
\end{aligned}
$$

where

(1) $P\{k \leq M+1, j \geq k, i>k\}=0$ because the case $i>k$ won't occur and $P(i>k)=0$;

(2) When $k>M+1$, at the $k$ th perfect inspection, the actual degradation level (equals to the inspected degradation level) is larger than both $d_{P M}$ and $d_{C M}$, that is to say $j$ can't be larger than $k$ and $P\{k>M+1, j>k\}=0$;

(3) When $k>M+1, j=k$, at the $k$ th perfect inspection, the actual degradation level (equals to the inspected degradation level) firstly exceeds both $d_{P M}$ and $d_{C M}$, that is to say $i$ equals to $k$ and $P\{k>M+1, j=k, i \neq k\}=0 ;$

(4) When $j \geq M+1, k>j$, at the $j$ th perfect inspection, the actual degradation level equals to the inspected degradation level and both firstly exceeds $d_{P M}$, that is to say $i$ equals to $j$ and $P\{j \geq M+1, k>j, i \neq j\}=0 ;$ 
In summary, by considering the distribution characteristic of system failure time $T_{F}$ and all possible values of the inspection number $j$ to conduct PM, and by summing over all the above cases and subcases, we can obtain the expected cost and length for policy $S_{B}$ as Equations (48) and (49). Therefore, the long run cost rate $C R_{B}$ for policy $S_{B}$ can be formulated by the ratio of $E\left(C_{B}\right)$ and $E\left(L_{B}\right)$, and the optimal $\tau, d_{P M}$ and $M$ for $S_{B}$ can be designed through minimizing $C R_{B}$.

$$
\begin{aligned}
E\left(C_{B}\right)= & \int_{0}^{+\infty} E\left(C_{B 1} \mid T_{F}\right) \cdot P_{B 1}\left(T_{F}\right) d\left[F_{T}(t)\right]+\sum_{j=1}^{+\infty} E\left(C_{B 2} \mid j\right) \cdot P_{B 2}(j) \\
& =\int_{0}^{M \tau}\left[E\left(C_{B 1.1} \mid T_{F}\right) \cdot P_{B 1.1}\left(T_{F}\right)+E\left(C_{B 1.2} \mid T_{F}\right) \cdot P_{B 1.2}\left(T_{F}\right)\right] d\left[F_{T}(t)\right] \\
& +\int_{M \tau}^{+\infty}\left[E\left(C_{B 1.3} \mid T_{F}\right) \cdot P_{B 1.3}\left(T_{F}\right)\right] d\left[F_{T}(t)\right] \\
& +\sum_{j=1}^{M}\left[E\left(C_{B 2.1} \mid j\right) \cdot P_{B 2.1}(j)+E\left(C_{B 2.2} \mid j\right) \cdot P_{B 2.2}(j)+E\left(C_{B 2.3} \mid j\right) \cdot P_{B 2.3}(j)\right] \\
& +\sum_{j=M+1}^{+\infty}\left[E\left(C_{B 2.4} \mid j\right) \cdot P_{B 2.4}(j)\right] \\
E\left(L_{B}\right)= & \int_{0}^{+\infty} E\left(L_{B 1} \mid T_{F}\right) \cdot P_{B 1}\left(T_{F}\right) d\left[F_{T}(t)\right]+\sum_{j=1}^{+\infty} E\left(L_{B 2} \mid j\right) \cdot P_{B 2}(j) \\
= & \int_{0}^{M \tau}\left[E\left(L_{B 1.1} \mid T_{F}\right) \cdot P_{B 1.1}\left(T_{F}\right)+E\left(L_{B 1.2} \mid T_{F}\right) \cdot P_{B 1.2}\left(T_{F}\right)\right] d\left[F_{T}(t)\right] \\
& +\int_{M \tau}^{+\infty}\left[E\left(L_{B 1.3} \mid T_{F}\right) \cdot P_{B 1.3}\left(T_{F}\right)\right] d\left[F_{T}(t)\right] \\
& +\sum_{j=1}^{M}\left[E\left(L_{B 2.1} \mid j\right) \cdot P_{B 2.1}(j)+E\left(L_{B 2.2} \mid j\right) \cdot P_{B 2.2}(j)+E\left(L_{B 2.3} \mid j\right) \cdot P_{B 2.3}(j)\right] \\
& +\sum_{j=M+1}^{+\infty}\left[E\left(L_{B 2.4} \mid j\right) \cdot P_{B 2.4}(j)\right]
\end{aligned}
$$

where $F_{T}(t)$ is also the CDF of the system failure time $T_{F}$, and can be referred to equation (26).

\section{Performance evaluation of the proposed policies}

In this section, we implement the two considered maintenance policies through numerical experiments and a real case study, and also illustrate their advantages over the classical maintenance policy with perfect inspections.

\subsection{Numerical experiments}

First, under the formulations of degradation model and maintenance policy in Section 2, we use a nonlinear gamma degradation process $\operatorname{Gamma}\left(1.5 \cdot t^{1.2}, 1.5\right)$ for illustration, and the other model and cost parameters are assumed to be $\sigma_{\varepsilon}=2, C_{I I}=1, C_{P I}=3, C_{P M}=30, C_{C M}=80$ and $d_{C M}=10$. Utilizing an iterated grid search (IGS) approach [45], optimization variables for policies $S_{A}$ and $S_{B}$ are obtained based on the cost analysis results in Sections 3.1 and 3.2, respectively. Then according to their optimal long run cost rate, the two policies are compared to the classical maintenance policy $S_{0}$, and their advantages are illustrated based on sensitivity 
analysis on the cost parameter, the variation of the degradation process and the measurement error.

For the proposed maintenance policy $S_{A}$ with imperfect inspections, we compute its long run cost rate $C R_{A}$, and plot the three-dimensional surface and the iso-level curve for $C R_{A}$ with respect to $\tau \in[1,2]$ and $d_{P M} \in[5,8]$, as shown in Figure 14, where the discretization steps of $\tau$ and $d_{P M}$ are respectively 0.01 and 0.1 . The surface is shown to be convex, which indicates that there is a global optimal combination of inspection and maintenance policy, i.e., $\tau=1.50$ and $d_{P M}=6.2$, corresponding to the minimum long run cost rate $C R_{A, \min }=7.53$. Furthermore, to increase the credibility of Figure 14, we fix one of the two variables $\tau, d_{P M}$, and present the values of $C R_{A}$ with respect to the other variable, as shown in Table 2 and Table 3.

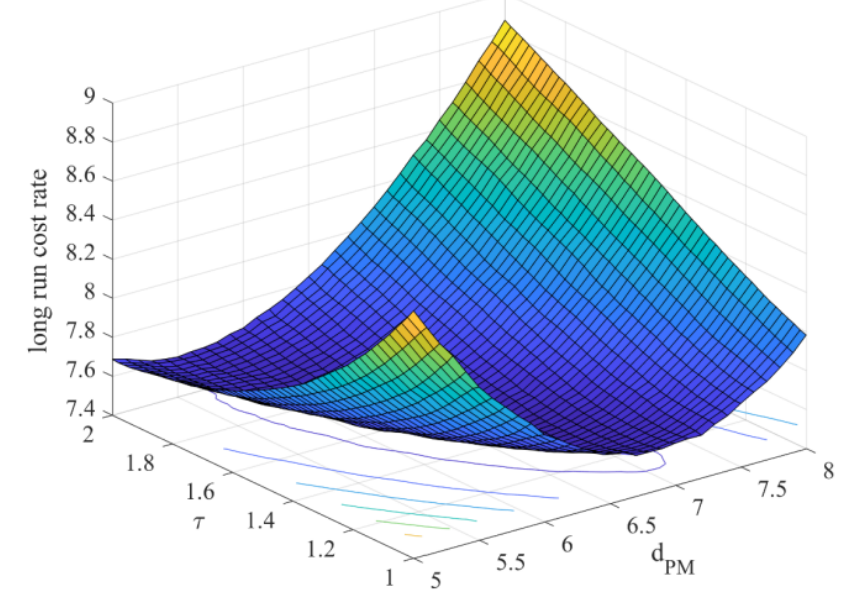

Figure 14. Long run cost rate $C R_{A}$ with respect to $\tau, d_{P M}$

Table 2. Long run cost rate $C R_{A}$ with respect to $\tau$ under fixed $d_{P M}=6.2$

\begin{tabular}{|c|c|c|c|c|c|c|c|c|c|c|c|}
\hline$\tau$ & 1.45 & 1.46 & 1.47 & 1.48 & 1.49 & 1.50 & 1.51 & 1.52 & 1.53 & 1.54 & 1.55 \\
\hline$C R_{B}$ & 7.557 & 7.552 & 7.543 & 7.539 & 7.537 & 7.534 & 7.535 & 7.536 & 7.538 & 7.540 & 7.542 \\
\hline
\end{tabular}

Table 3. Long run cost rate $C R_{A}$ with respect to $d_{P M}$ under fixed $\tau=1.50$

\begin{tabular}{|c|c|c|c|c|c|c|c|c|c|c|c|}
\hline$d_{P M}$ & 6 & 6.1 & 6.2 & 6.3 & 6.4 & 6.5 & 6.6 & 6.7 & 6.8 & 6.9 & 7 \\
\hline$C R_{B}$ & 7.56 & 7.54 & 7.53 & 7.54 & 7.54 & 7.56 & 7.57 & 7.59 & 7.62 & 7.65 & 7.69 \\
\hline
\end{tabular}

For the proposed maintenance policy $S_{B}$ with two-stage inspection scheme, its long run cost rate $C R_{B}$ can be obtained by the ratio of $E\left(C_{B}\right)$ and $E\left(L_{B}\right)$. By computing $C R_{B}$ under different values of $\tau, d_{P M}$ and $M$, the minimum long run cost rate is found to be $C R_{\mathrm{B}, \min }=7.42$, corresponding to the optimization variables $\tau=1.47, d_{P M}=6.5$ and $M=4$. Furthermore, to visually show how $C R_{B}$ changes with $\tau, d_{P M}$ and $M$, we fix $M$ to be its optimal value 4, and plot the three-dimensional surface for $C R_{B}$ with respect to $\tau \in[1,2]$ and $d_{P M} \in[5.5,8]$, where the discretization steps of $\tau$ and $d_{P M}$ are respectively 0.01 and 0.1 . Besides, the values of 
$C R_{B}$ with respect to one of the two variables $\tau, d_{P M}$, by fixing the other variable, are presented in Table 4 and Table 5. Similar to the plot of $C R_{A}$ in Figure 14, the surface for $C R_{B}$ in Figure 15 is also convex, indicating the existence of global optimization for policy $S_{B}$.

Table 4. Long run cost rate $C R_{B}$ with respect to $\tau$ under fixed $d_{P M}=6.5, M=4$

\begin{tabular}{|c|c|c|c|c|c|c|c|c|c|c|c|}
\hline$\tau$ & 1.40 & 1.41 & 1.42 & 1.43 & 1.44 & 1.45 & 1.46 & 1.47 & 1.48 & 1.49 & 1.50 \\
\hline$C R_{B}$ & 7.434 & 7.432 & 7.429 & 7.427 & 7.425 & 7.422 & 7.420 & 7.421 & 7.423 & 7.425 & 7.427 \\
\hline
\end{tabular}

Table 5. Long run cost rate $C R_{B}$ with respect to $d_{P M}$ under fixed $\tau=1.47, M=4$

\begin{tabular}{|c|c|c|c|c|c|c|c|c|c|c|c|}
\hline$d_{P M}$ & 6 & 6.1 & 6.2 & 6.3 & 6.4 & 6.5 & 6.6 & 6.7 & 6.8 & 6.9 & 7 \\
\hline$C R_{B}$ & 7.52 & 7.49 & 7.47 & 7.45 & 7.43 & 7.42 & 7.43 & 7.43 & 7.44 & 7.44 & 7.47 \\
\hline
\end{tabular}

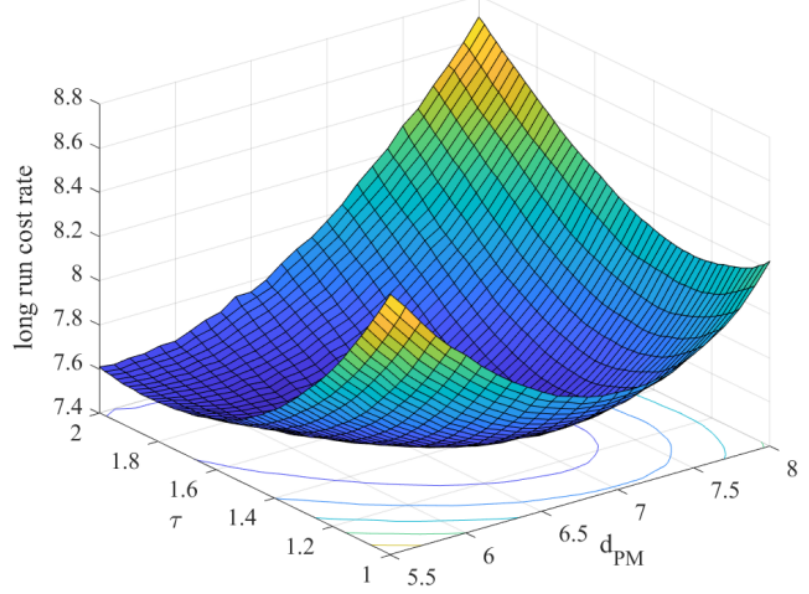

Figure 15. Long run cost rate $C R_{B}$ with respect to $\tau, d_{P M}$ under fixed $M=4$

In addition, under fixed $\tau=1.47$ and $d_{P M}=6.5$, the values of $C R_{B}$ with respect to $M \in[1,10]$ is provided in Table 6, and the changing curve is plotted in Figure 16, where the discretization steps of $M$ is 1 . It can be seen that $C R_{B}$ decreases when $M$ increases from 1 to 4, then increases with $M$ and finally converges to 7.57 when $M$ is larger than 6, which is because that for the system under maintenance policy $S_{B}$ with large enough $M$, the occurrence of failure will be earlier than the shifting time of the inspections from imperfect ones to perfect ones, and the system maintenance will have nothing to do with $M$.

Table 6. Long run cost rate $C R_{B}$ with respect to $M$ under fixed $\tau=1.47, d_{P M}=6.5$

\begin{tabular}{|c|c|c|c|c|c|c|c|c|c|c|}
\hline$M$ & 1 & 2 & 3 & 4 & 5 & 6 & 7 & 8 & 9 & 10 \\
\hline$C R_{B}$ & 7.99 & 7.68 & 7.49 & 7.42 & 7.51 & 7.56 & 7.57 & 7.57 & 7.57 & 7.57 \\
\hline
\end{tabular}




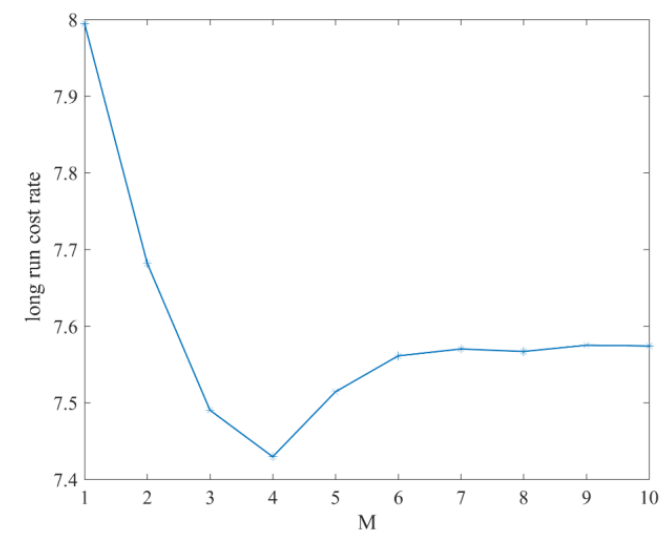

Figure 16. Long run cost rate $C R_{B}$ with respect to $M$ under fixed $\tau=1.47, d_{P M}=6.5$

\subsection{Sensitivity analysis}

Based on different perfect inspection cost $C_{P I}$, the optimal long run cost rates of the three policies are plotted in Figure 17. It can be shown that with the increase of $C_{P I}$, the optimal cost rate of policies $S_{0}$ and $S_{B}$ rise obviously, while policy $S_{A}$ is not affected. The curves for policies $S_{0}$ and $S_{A}$ cross at a point corresponding to when perfect inspections are not too cheap and $C_{P I}$ is higher than about 2.6, $S_{A}$ will be more economical than $S_{0}$. Besides, for all values of $C_{P I}$, policy $S_{B}$ is always the best choice in that it approaches the curve of $S_{0}$ when $C_{P I}$ is low, and converges to $S_{A}$ when $C_{P I}$ is high.

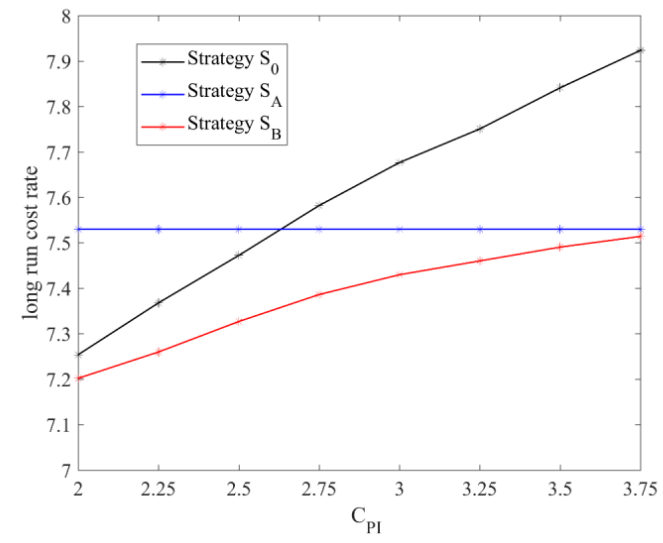

Figure 17. Sensitivity analysis of the optimal maintenance cost rate to $C_{P I}$

In addition, we also investigate the effect of PM and $\mathrm{CM}$ cost on the maintenance policies, as shown in Figures 18 and 19, respectively. It can be seen that as $C_{P M}$ or $C_{C M}$ increases, the curves of all three policies show obvious rising trends, and in an economical viewpoint, policy $S_{B}$ is always the best and $S_{0}$ is the worst. Besides, the sensitivity on $C_{P M}$ is larger than that on $C_{C M}$, which is because that all three CBM strategies are meant to prevent the occurrence of failures, therefore is less influenced by the cost incurred by $\mathrm{CM}$ activities against failures. 


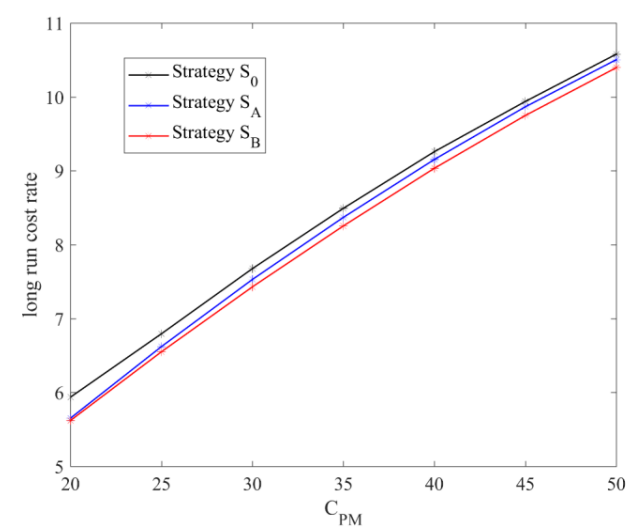

Figure 18. Sensitivity analysis of the optimal maintenance cost rate to $C_{P M}$

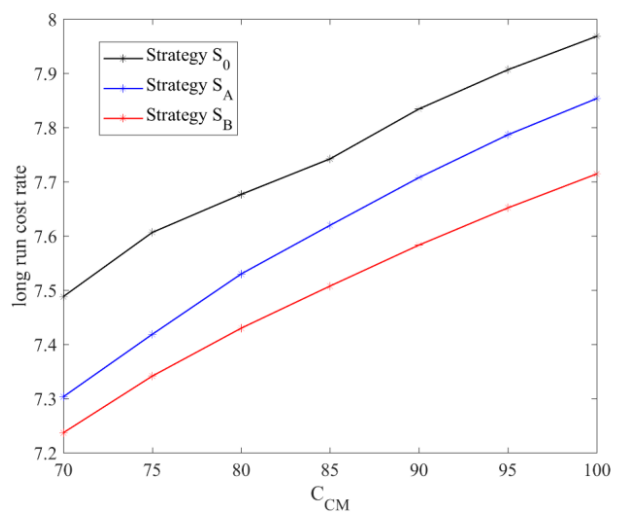

Figure 19. Sensitivity analysis of the optimal maintenance cost rate to $C_{C M}$

Additionally, considering that the variation of the degradation process and the measurement error can affect the maintenance policies, we plot the sensitivity analysis of the optimal maintenance cost rate to $\alpha$ and $\sigma_{\varepsilon}$ in Figures 20 and 21. Firstly, in order to keep the comparability of policies with different values of $\alpha$, we fix the form of nonlinear drift function $\Lambda(t)$ and the mean function of the degradation process $\frac{\alpha}{\beta} \Lambda(t)$. Under this circumstance, if we increase the value of $\alpha$, we also have to incarease that of $\beta$ proportionally, and this will lead to smaller variation of the degradation process, i.e., $\frac{\alpha}{\beta^{2}} \Lambda(t)$, and decrease the optimal cost rates for all the three policies, although in different rates. Besides, the increase of $\sigma_{\varepsilon}$ will make it harder to prevent the occurrence of failure, and result in higher cost rates for policies $S_{A}$ and $S_{B}$. For the comparison among the three policies, we can see that policy $S_{B}$ still has the best economic performance for all values of $\alpha$ and $\sigma_{\varepsilon}$, and policy $S_{0}$ turns to be worse than $S_{A}$ when $\alpha$ is smaller than about 2.2, or when $\sigma_{\varepsilon}$ exceeds around 2.25 . 


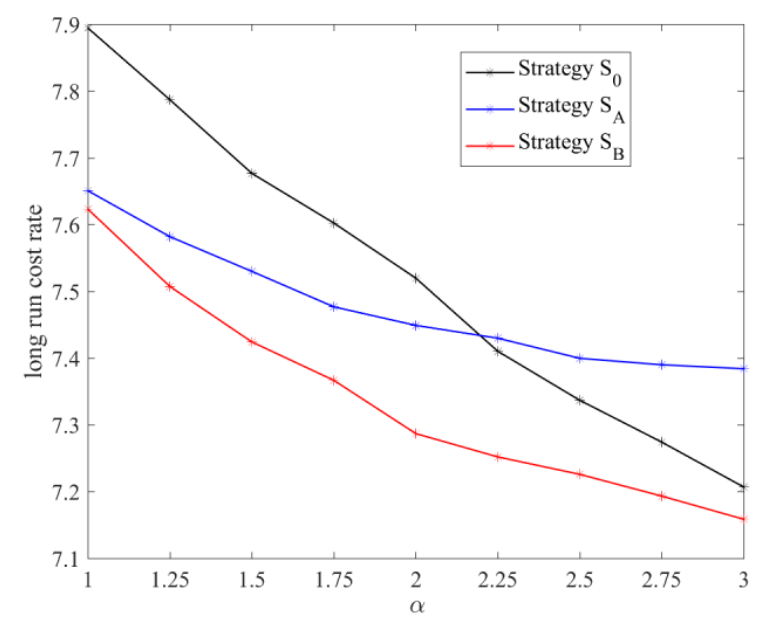

Figure 20. Sensitivity analysis of the optimal maintenance cost rate to $\alpha$

Figure 21 gives a good insight into the adaptive behavior of policy $S_{B}$. Since it mixes both perfect and imperfect inspection so that it can take the best of both kind of inspections: inspecting at low cost when accuracy is not highly required and using perfect inspections when the accurate information on the degradation level becomes critical. When compared to the two other policies $S_{0}$ and $S_{A}$, policy $S_{B}$ allows substantial savings in situations where the quality/cost ratio of imperfect inspections does not take extreme values: in this case, it is highly relevant to resort to both perfect and imperfect inspections within the same policy. In extreme situations, Figure 21 clearly shows that the best option is to use either only perfect inspections (when imperfect inspection are too expensive for their quality, i.e. on the right of the figure and in this case policy $S_{B}$ becomes equivalent to policy $S_{0}$ ) or only imperfect inspections (when imperfect inspections are of high quality and not expensive, i.e. on the left of the figure, and in this case policy $S_{B}$ becomes equivalent to policy $S_{A}$ ). Because of its versatility, policy $S_{B}$ can thus be adapted to all the configurations and outperform both policies $S_{0}$ and $S_{A}$ in all the cases.

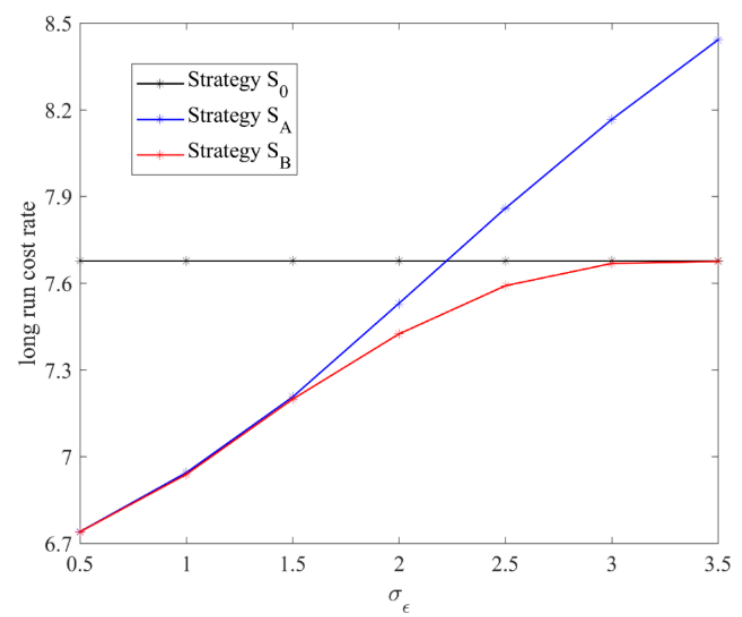

Figure 21. Sensitivity analysis of the optimal maintenance cost rate to $\sigma_{\varepsilon}$ 


\subsection{Robustness Analysis}

It should be noted that the above analysis is all based on the assumption of an accurate knowledge about inspection quality. However, in practice we may mis-specify the model assumptions, e.g. on whether the inspections are perfect or not, and on the exact value of the variance of measurement errors. Therefore, we analyze the robustness of the three maintenance policies by studying the effect of inspection quality mis-specification. In Tables 7-9, the optimal long run cost rates are evaluated by implementing on a system with the measurement error standard deviation at its true $\sigma_{\varepsilon}$ value, and the maintenance policies as well as decision variables optimized for wrongly specified $\sigma_{\varepsilon}$.

As the deviation between the true and wrongly specified $\sigma_{\varepsilon}$ increases, the optimal long run cost rates for the three policies all increase. Besides, the proposed policies $S_{A}$ and $S_{B}$ are always better than the classical policy $S_{0}$ for all true and wrongly specified $\sigma_{\varepsilon}$. For the comparison between $S_{A}$ and $S_{B}$, it can be indicated that when the true $\sigma_{\varepsilon}$ is bigger than the wrongly specified $\sigma_{\varepsilon}$, i.e., when we overestimate the measurement instrument performance and underestimate the measurement error, then it is more beneficial to adopt the maintenance policy $S_{B}$. When the true $\sigma_{\varepsilon}$ is smaller than the wrongly specified $\sigma_{\varepsilon}$, i.e., when we underestimate the measurement instrument performance and overestimate the size of measurement error, then maintenance policy $S_{A}$ is more preferable from an economic point of view. One of the interesting conclusion of this analysis is about the robustness of the proposed policies $S_{A}$ and $S_{B}$ : even if a wrong value of the measurement error variance is used to optimize these policies, they are robust enough so that their performance remains better than the policy $S_{0}$ that assumes wrongly perfect inspections.

Table 7. Optimal long run cost rate for mis-specified policy $S_{0}$

\begin{tabular}{|c|c|}
\hline True $\sigma_{\varepsilon} \quad$ Wror & 0 \\
\hline 0.5 & 7.68 \\
\hline 1 & 7.76 \\
\hline 1.5 & 7.94 \\
\hline 2 & 8.22 \\
\hline 2.5 & 8.52 \\
\hline 3 & 8.81 \\
\hline 3.5 & 9.06 \\
\hline
\end{tabular}

Table 8. Optimal long run cost rate for mis-specified policy $S_{A}$

\begin{tabular}{|c|c|c|c|c|c|c|c|}
\hline $\begin{array}{lll} & \text { Wrongly specified } \sigma_{\varepsilon} \\
\text { True } \sigma_{\varepsilon} & \end{array}$ & 0.5 & 1 & 1.5 & 2 & 2.5 & 3 & 3.5 \\
\hline 0.5 & 6.74 & 6.75 & 6.76 & 6.75 & 6.76 & 6.78 & 6.79 \\
\hline
\end{tabular}




\begin{tabular}{|c|c|c|c|c|c|c|c|}
\hline 1 & 6.95 & $\mathbf{6 . 9 4}$ & 6.94 & 6.95 & 6.96 & 7.01 & 7.01 \\
\hline 1.5 & 7.23 & 7.22 & $\mathbf{7 . 2 1}$ & 7.22 & 7.25 & 7.29 & 7.29 \\
\hline 2 & 7.54 & 7.54 & 7.53 & $\mathbf{7 . 5 3}$ & 7.55 & 7.59 & 7.60 \\
\hline 2.5 & 7.89 & 7.89 & 7.88 & 7.87 & $\mathbf{7 . 8 6}$ & 7.89 & 7.90 \\
\hline 3 & 8.21 & 8.2 & 8.22 & 8.2 & 8.2 & $\mathbf{8 . 1 7}$ & 8.19 \\
\hline 3.5 & 8.53 & 8.51 & 8.54 & 8.49 & 8.51 & 8.46 & $\mathbf{8 . 4 4}$ \\
\hline
\end{tabular}

Table 9. Optimal long run cost rate for mis-specified policy $S_{B}$

\begin{tabular}{|c|c|c|c|c|c|c|c|}
\hline $\begin{array}{lll} & \text { Wrongly specified } \sigma_{\varepsilon} \\
\text { True } \sigma_{\varepsilon} & \end{array}$ & 0.5 & 1 & 1.5 & 2 & 2.5 & 3 & 3.5 \\
\hline 0.5 & 6.74 & 6.75 & 6.76 & 7.00 & 7.25 & 7.67 & 7.68 \\
\hline 1 & 6.95 & 6.94 & 6.95 & 7.10 & 7.29 & 7.67 & 7.68 \\
\hline 1.5 & 7.23 & 7.22 & 7.20 & 7.25 & 7.35 & 7.67 & 7.68 \\
\hline 2 & 7.53 & 7.53 & 7.52 & 7.32 & 7.45 & 7.67 & 7.68 \\
\hline 2.5 & 7.86 & 7.87 & 7.87 & 7.65 & 7.59 & 7.67 & 7.68 \\
\hline 3 & 8.19 & 8.19 & 8.18 & 7.88 & 7.78 & 7.67 & 7.68 \\
\hline 3.5 & 8.50 & 8.51 & 8.52 & 8.12 & 7.96 & 7.67 & 7.68 \\
\hline
\end{tabular}

\subsection{Case study}

In order to show how the presented maintenance policies can be used in practical applications, we apply them to a "three-bladed rotor system" on an offshore wind turbine. A blade is an important part of a wind turbine, and its failure is caused by residual strength reduction due to crack growth [46]. In fact, cracks on blades are usually monitored by periodic inspections, but such crack length measurements by imperfect inspection systems are often contaminated by errors [47]. Therefore, this case study is intended to implement the two proposed maintenance policies with imperfect inspections, and to see which policy offers the best benefit.

The dataset used in this study is referred to some relevant references $[48,49]$. The blade crack growth process can be modelled by a homogeneous gamma process with shape and scale parameters given by $\alpha=0.542 \mathrm{~cm} /$ month and $\beta=1.147$. Therefore, the mean degradation rate will be $\alpha / \beta=0.4725 \mathrm{~cm} /$ month. In addition, the failure threshold level for the blade crack length is $d_{C M}=20 \mathrm{~cm}$. For the imperfectly inspected crack length, the variation of the measurement error is $\sigma_{\varepsilon}=1 \mathrm{~cm}$. As for the cost for the blades, the cost for each perfect inspection is $C_{P I}=3000 €$, the cost for each imperfect inspection is $C_{I I}=2000 €$, the Cost of performing a corrective maintenance is $C_{C M}=440400 €$, and the Cost of performing a preventive maintenance is $C_{P M}=225000 €$.

Also utilizing an iterated grid search (IGS) approach, we obtain optimization variables for the classical maintenance policy $S_{0}$, the proposed maintenance policies $S_{A}$ and $S_{B}$, respevtively. Table 10 lists the values of $\tau, d_{P M}, M$ as well as the the corresponding long run cost rate and the percentage reduction of policies $S_{A}$ and $S_{B}$ compared to policy $S_{0}$. It can be indicated that for the "three-bladed rotor system" on an offshore wind turbine, making maintenance decisions with 
imperfect inspections allows substantial savings than making decisions with perfect ones, and it is more economically beneficial to mix both perfect and imperfect inspections, and adapt the more flexible maintenance policy $S_{B}$.

Table 10. A comparison between the three maintenance policies for the blade case

\begin{tabular}{|c|c|c|c|c|c|}
\hline $\begin{array}{c}\text { Maintenance } \\
\text { policy }\end{array}$ & $\begin{array}{c}\text { optimal } \tau \\
\text { (month) }\end{array}$ & $\begin{array}{c}\text { optimal } \\
d_{P M}(\mathrm{~cm})\end{array}$ & $\begin{array}{c}\text { optimal } \\
M\end{array}$ & $\begin{array}{c}\text { Minimum long } \\
\text { run cost rate } € \\
/ \text { month) }\end{array}$ & $\begin{array}{c}\text { Cost reduction } \\
\text { compared to pocily } \\
S_{0}\end{array}$ \\
\hline$S_{0}$ & 6.2 & 16 & - & 6980.20 & 0 \\
\hline$S_{A}$ & 5.7 & 16 & - & 6918.97 & $0.88 \%$ \\
\hline$S_{B}$ & 5.3 & 16 & 6 & 6881.87 & $1.41 \%$ \\
\hline
\end{tabular}

Furthermore, to account for other potential crack length inspection systems, we analyze the most economical maintenance policy $S_{B}$ in different cases of imperfect inspection cost and variation of measurement error, and list the corresponding minimum long run cost rates in Table 11. It can be indicated that compared to the case with medium values of $C_{I I}$ and $\sigma_{\varepsilon}$, the economic performance is better in the case with lower imperfect inspection cost and larger measurement error. Therefore, the wind turbine operators are advised to make more efforts on decreasing the cost of an imperfect inspection system, even by paying the price of inspection quality.

Table 11. A comparison for maintenance policy $S_{B}$ with different values of $C_{I I}$ and $\sigma_{\varepsilon}$

\begin{tabular}{|c|c|c|c|}
\hline $\begin{array}{c}\text { Imperfect inspection } \\
\text { cost } C_{I I}(€)\end{array}$ & $\begin{array}{c}\text { Variation of } \\
\text { measurement error } \\
(\mathrm{cm})\end{array}$ & $\begin{array}{c}\text { Minimum long run } \\
\text { cost rate }(€ / \text { month })\end{array}$ & $\begin{array}{c}\text { Cost reduction } \\
\text { compared to pocily } \\
S_{0}\end{array}$ \\
\hline 2000 & 1 & 6881.87 & $1.41 \%$ \\
\hline 1800 & 1.1 & 6845.37 & $1.93 \%$ \\
\hline 2200 & 0.9 & 6899.31 & $1.16 \%$ \\
\hline
\end{tabular}

\section{Conclusion}

Considering the measurement errors for the inspections of continuous degradation processes, this paper proposes two CBM policies $S_{A}$ and $S_{B}$. Maintenance decisions of them are respectively made upon a purely imperfect inspection scheme and a two-stage inspection scheme, i.e., to shift from imperfect inspections to perfect ones after $M$ inspections. To calculate the long run cost rates of the two policies, we derive the occurrence probabilities of conditional events concerning TP, TN, FP and FN, and compute the occurrence probabilities, expected cost and length for the renewal cycle in different cases. The two policies are implemented through some numerical experiments, and sensitivity analysis shows that the proposed policy $S_{A}$ is more economical than the classical one $S_{0}$ on the condition that the perfect inspection cost is not too low, variation of the degradation process is not too small or the size of measurement error is not too large. Besides, thanks to the generalization introduced by two-stage inspection scheme, the proposed policy $S_{B}$ always has the best economic performance among the three maintenance policies, and is recommended to be chosen in practice. In addition, by analyzing the effect of inspection quality mis-specification on 
the maintenance policies, the proposed policies $S_{A}$ and $S_{B}$ are found to be more robust than $S_{0}$. Furthermore, a real case study of a "three-bladed rotor system" on an offshore wind turbine is presented to show how the proposed maintenance policies can be used in practical applications.

Our model and our results can give some insight on how different kinds of inspections, each with different quality and different costs, should be optimally used and sequenced to take the most benefit of them. once implemented within computer-aided maintenance management system, our proposed model would be hence of interest to maintenance decision-makers: as a stationary control limit rule, the proposed model is easy to implement. Besides, the mixture of perfect and imperfect inspections lead to an most beneficial system monitoring, and the amount of cost savings obviously depend on the different costs of the different maintenance actions, inspections and on the different features of the maintenance actions and inspections available for a given maintained system.

The model of imperfect inspection could be further improved in several ways, which opens several research perspectives. Firstly, the dependence between the variance of the inspection noise and the measured deterioration level should be considered to make the maintenance policy more realistic. Besides, the proposed model can be generalized by taking into account imperfect maintenance activities. Furthermore, another interesting work can be the issue of maintenance decision-making using different inspections with different quality and cost characteristics.

\section{Acknowledgement}

This work was supported by the National Natural Science Foundation of China under Grant 71672006, the Grant JSZL2017601B006, and the Fundamental Research Funds for the Central Universities under Grant No. YWF-19-BJ-J-160.

\section{References}

[1] A.F. Shahraki, O.P. Yadav, H. Liao, A Review on Degradation Modelling and Its Engineering Applications, Int. J. Perform. Eng. 13 (2017) 299-314.

[2] D.S. González-González, R.J. Praga-Alejo, M. Cantú-Sifuentes, A non-linear fuzzy degradation model for estimating reliability of a polymeric coating, Appl. Math. Model. 40 (2016) 1387-1401. https://doi.org/10.1016/j.apm.2015.06.033.

[3] Z.-S. Ye, M. Xie, Stochastic modelling and analysis of degradation for highly reliable products, Appl. Stoch. Models Bus. Ind. 31 (2015) 16-32. https://doi.org/10.1002/asmb.2063.

[4] D. Pan, J.-B. Liu, F. Huang, J. Cao, A. Alsaedi, A Wiener process model with truncated normal distribution for reliability analysis, Appl. Math. Model. 50 (2017) 333-346. https://doi.org/10.1016/j.apm.2017.05.049.

[5] S. Hao, J. Yang, C. Berenguer, Nonlinear step-stress accelerated degradation modelling considering three sources of variability, Reliab. Eng. Syst. Saf. 172 (2018) 207-215. https://doi.org/10.1016/j.ress.2017.12.012.

[6] G. Pulcini, A perturbed gamma process with statistically dependent measurement errors, Reliab. Eng. Syst. Saf. 152 (2016) 296-306. https://doi.org/10.1016/j.ress.2016.03.024.

[7] C. Paroissin, Online Estimation Methods for the Gamma Degradation Process, IEEE Trans. Reliab. 66 (2017) 1361-1367. https://doi.org/10.1109/TR.2017.2757768.

[8] D. He, Y. Wang, G. Chang, Objective Bayesian analysis for the accelerated degradation model based on the inverse Gaussian process, Appl. Math. Model. 61 (2018) 341-350. https://doi.org/10.1016/j.apm.2018.04.025.

[9] J. Guo, C. Wang, J. Cabrera, E.A. Elsayed, Improved inverse Gaussian process and bootstrap: Degradation and reliability metrics, Reliab. Eng. Syst. Saf. 178 (2018) 269-277. 
https://doi.org/10.1016/j.ress.2018.06.013.

[10] A. Grall, C. Bérenguer, L. Dieulle, A condition-based maintenance policy for stochastically deteriorating systems, Reliab. Eng. Syst. Saf. 76 (2002) 167-180. https://doi.org/10.1016/S0951-8320(01)00148-X.

[11] B. de Jonge, R. Teunter, T. Tinga, The influence of practical factors on the benefits of condition-based maintenance over time-based maintenance, Reliab. Eng. Syst. Saf. 158 (2017) 2130. https://doi.org/10.1016/j.ress.2016.10.002.

[12] R. Ahmad, S. Kamaruddin, An overview of time-based and condition-based maintenance in industrial application, Comput. Ind. Eng. 63 (2012) 135-149. https://doi.org/10.1016/j.cie.2012.02.002.

[13] J.M. van Noortwijk, A survey of the application of gamma processes in maintenance, Reliab. Eng. Syst. Saf. 94 (2009) 2-21. https://doi.org/10.1016/j.ress.2007.03.019.

[14] S. Alaswad, Y. Xiang, A review on condition-based maintenance optimization models for stochastically deteriorating system, Reliab. Eng. Syst. Saf. 157 (2017) 54-63. https://doi.org/10.1016/j.ress.2016.08.009.

[15] Z. Zhang, X. He, A. Kusiak, Data-driven minimization of pump operating and maintenance cost, Eng. Appl. Artif. Intell. 40 (2015) 37-46. https://doi.org/10.1016/j.engappai.2015.01.003.

[16] M. Baptista, S. Sankararaman, Ivo.P. de Medeiros, C. Nascimento, H. Prendinger, E.M.P. Henriques, Forecasting fault events for predictive maintenance using data-driven techniques and ARMA modeling, Comput. Ind. Eng. 115 (2018) 41-53. https://doi.org/10.1016/j.cie.2017.10.033.

[17] H. Liao, E.A. Elsayed, L.-Y. Chan, Maintenance of continuously monitored degrading systems, Eur. J. Oper. Res. 175 (2006) 821-835. https://doi.org/10.1016/j.ejor.2005.05.017.

[18] A.K.S. Jardine, D. Lin, D. Banjevic, A review on machinery diagnostics and prognostics implementing condition-based maintenance, Mech. Syst. Signal Process. 20 (2006) 1483-1510. https://doi.org/10.1016/j.ymssp.2005.09.012.

[19] V. Babishin, S. Taghipour, Optimal maintenance policy for multicomponent systems with periodic and opportunistic inspections and preventive replacements, Appl. Math. Model. 40 (2016) 10480-10505. https://doi.org/10.1016/j.apm.2016.07.019.

[20] K.T. Huynh, A. Barros, C. Bérenguer, I.T. Castro, A periodic inspection and replacement policy for systems subject to competing failure modes due to degradation and traumatic events, Reliab. Eng. Syst. Saf. 96 (2011) 497-508. https://doi.org/10.1016/j.ress.2010.12.018.

[21] B. Castanier, A. Grall, C. Bérenguer, A condition-based maintenance policy with nonperiodic inspections for a two-unit series system, Reliab. Eng. Syst. Saf. 87 (2005) 109-120. https://doi.org/10.1016/j.ress.2004.04.013.

[22] K.T.P. Nguyen, M. Fouladirad, A. Grall, New Methodology for Improving the Inspection Policies for Degradation Model Selection According to Prognostic Measures, IEEE Trans. Reliab. 67 (2018) 1269-1280. https://doi.org/10.1109/TR.2018.2829738.

[23] Z. Zhang, X. Si, C. Hu, Y. Lei, Degradation data analysis and remaining useful life estimation: A review on Wiener-process-based methods, Eur. J. Oper. Res. 271 (2018) 775-796. https://doi.org/10.1016/j.ejor.2018.02.033.

[24] Q. Zhai, Z.-S. Ye, J. Yang, Y. Zhao, Measurement errors in degradation-based burn-in, Reliab. Eng. Syst. Saf. 150 (2016) 126-135. https://doi.org/10.1016/j.ress.2016.01.015.

[25] X.-S. Si, M.-Y. Chen, W. Wang, C.-H. Hu, D.-H. Zhou, Specifying measurement errors for required lifetime estimation performance, Eur. J. Oper. Res. 231 (2013) 631-644. https://doi.org/10.1016/j.ejor.2013.05.046.

[26] Z.-X. Zhang, X.-S. Si, C.-H. Hu, Q. Zhang, T.-M. Li, C.-Q. Xu, Planning Repeated Degradation Testing for Products With Three-Source Variability, IEEE Trans. Reliab. 65 (2016) 640-647. https://doi.org/10.1109/TR.2015.2512223.

[27] B. de Jonge, P.A. Scarf, A review on maintenance optimization, Eur. J. Oper. Res. (2019). https://doi.org/10.1016/j.ejor.2019.09.047.

[28] M.D. Berrade, C.A.V. Cavalcante, P.A. Scarf, Maintenance scheduling of a protection system subject to imperfect inspection and replacement, Eur. J. Oper. Res. 218 (2012) 716-725. https://doi.org/10.1016/j.ejor.2011.12.003.

[29] M.D. Berrade, P.A. Scarf, C.A.V. Cavalcante, Some Insights Into the Effect of Maintenance Quality for a Protection System, IEEE Trans. Reliab. 64 (2015) 661-672. https://doi.org/10.1109/TR.2015.2417431. 
[30] A. Barros, A. Grall, C. Berenguer, Joint modelling and optimization of monitoring and maintenance performance for a two-unit parallel system, Proc. Inst. Mech. Eng. Part O J. Risk Reliab. 221 (2007) 1-11. https://doi.org/10.1243/1748006XJRR31.

[31] X.-Y. Li, H.-Z. Huang, Y.-F. Li, E. Zio, Reliability assessment of multi-state phased mission system with non-repairable multi-state components, Applied Mathematical Modelling. 61 (2018) 181-199. https://doi.org/10.1016/j.apm.2018.04.008.

[32] M.D. Berrade, A two-phase inspection policy with imperfect testing, Appl. Math. Model. 36 (2012) 108-114. https://doi.org/10.1016/j.apm.2011.05.035.

[33] M.D. Berrade, P.A. Scarf, C.A.V. Cavalcante, R.A. Dwight, Imperfect inspection and replacement of a system with a defective state: A cost and reliability analysis, Reliab. Eng. Syst. Saf. 120 (2013) 80-87. https://doi.org/10.1016/j.ress.2013.02.024.

[34] J.P.C. Driessen, H. Peng, G.J. van Houtum, Maintenance optimization under non-constant probabilities of imperfect inspections, Reliab. Eng. Syst. Saf. 165 (2017) 115-123. https://doi.org/10.1016/j.ress.2017.03.020.

[35] A.R. Alberti, C.A.V. Cavalcante, P. Scarf, A.L.O. Silva, Modelling inspection and replacement quality for a protection system, Reliab. Eng. Syst. Saf. 176 (2018) 145-153. https://doi.org/10.1016/j.ress.2018.04.002.

[36] G. Levitin, L. Xing, H.-Z. Huang, Cost effective scheduling of imperfect inspections in systems with hidden failures and rescue possibility, Appl. Math. Model. 68 (2019) 662-674. https://doi.org/10.1016/j.apm.2018.12.001.

[37] B. Liu, X. Zhao, R.-H. Yeh, W. Kuo, Imperfect Inspection Policy for Systems with Multiple Correlated Degradation Processes, IFAC-Pap. 49 (2016) 1377-1382. https://doi.org/10.1016/j.ifacol.2016.07.758.

[38] J.T. Yoon, B.D. Youn, M. Yoo, Y. Kim, S. Kim, Life-cycle maintenance cost analysis framework considering time-dependent false and missed alarms for fault diagnosis, Reliab. Eng. Syst. Saf. 184 (2019) 181-192. https://doi.org/10.1016/j.ress.2018.06.006.

[39] K.T. Huynh, A. Barros, C. Bérenguer, Adaptive condition-based maintenance decision framework for deteriorating systems operating under variable environment and uncertain condition monitoring, Proc. Inst. Mech. Eng. Part O J. Risk Reliab. 226 (2012) 602-623. https://doi.org/10.1177/1748006X12465718.

[40] J. Shen, L. Cui, Optimal scheduling of imperfect and perfect inspections for systems subject to continuous degradation, in: 2017 IEEE Int. Conf. Ind. Eng. Eng. Manag. IEEM, IEEE, Singapore, 2017: pp. 1940-1944. https://doi.org/10.1109/IEEM.2017.8290230.

[41] K.T.P. Nguyen, P. Do, K.T. Huynh, C. Bérenguer, A. Grall, Joint optimization of monitoring quality and replacement decisions in condition-based maintenance, Reliab. Eng. Syst. Saf. 189 (2019) 177-195. https://doi.org/10.1016/j.ress.2019.04.034.

[42] J.M. van Noortwijk, J.A.M. van der Weide, Applications to continuous-time processes of computational techniques for discrete-time renewal processes, Reliab. Eng. Syst. Saf. 93 (2008) 1853-1860. https://doi.org/10.1016/j.ress.2008.03.023.

[43] H.C. Tijms, A first course in stochastic models, John Wiley and sons, 2003.

[44] Park, K. S, Optimal continuous-wear limit replacement under periodic inspections, IEEE Trans. Reliab. 37 (1988) 97-102. https://doi.org/10.1109/24.3722.

[45] A.S. Ramkumar, S.G. Ponnambalam, N. Jawahar, A new iterated fast local search heuristic for solving QAP formulation in facility layout design, Robot. Comput.-Integr. Manuf. 25 (2009) 620-629. https://doi.org/10.1016/j.rcim.2008.03.022.

[46] W. Zhu, M. Fouladirad, C. Bérenguer, A predictive maintenance policy based on the blade of offshore wind turbine, in: 2013 Proc. Annu. Reliab. Maintainab. Symp. RAMS, 2013: pp. 1-6. https://doi.org/10.1109/RAMS.2013.6517637.

[47] M.J. Kallen, J.M. van Noortwijk, Optimal maintenance decisions under imperfect inspection, Reliab. Eng. Syst. Saf. 90 (2005) 177-185. https://doi.org/10.1016/j.ress.2004.10.004.

[48] F. Besnard, L. Bertling, An Approach for Condition-Based Maintenance Optimization Applied to Wind Turbine Blades, IEEE Trans. Sustain. Energy. 1 (2010) 77-83. https://doi.org/10.1109/tste.2010.2049452.

[49] M. Shafiee, M. Finkelstein, C. Bérenguer, An opportunistic condition-based maintenance policy for offshore wind turbine blades subjected to degradation and environmental shocks, Reliab. Eng. Syst. Saf. 142 (2015) 463-471. https://doi.org/10.1016/j.ress.2015.05.001. 


\section{Appendix}

The occurrence probabilities of other possible conditional events for two successive inspection results are presented as follows:

$$
\begin{aligned}
& P\left(X_{n} \geq d_{C M} \mid T N_{n-1}\right)=P\left\{X_{k} \geq d_{C M} \mid X_{n-1} \leq d_{P M}, Z_{n-1} \leq d_{P M}\right\} \\
& =\frac{\int_{0}^{d_{P M}}\left[1-F_{\Delta X_{n-1, n}}\left(d_{C M}-x\right)\right] \cdot \Phi\left(\frac{d_{P M}-x}{\sigma_{\varepsilon}}\right) f_{X_{n-1}}(x) d x}{\int_{0}^{d_{P M}} \Phi\left(\frac{d_{P M}-x}{\sigma_{\varepsilon}}\right) f_{X_{n-1}}(x) d x} \\
& P\left(F N_{n} \mid T N_{n-1}\right)=P\left\{d_{P M}<X_{n} \leq d_{C M}, Z_{n} \leq d_{P M} \mid X_{n-1} \leq d_{P M}, Z_{n-1} \leq d_{P M}\right\} \\
& =\frac{\int_{0}^{d_{P M}} \int_{d_{P M}-x}^{d_{C M}-x} \Phi\left(\frac{d_{P M}-x-y}{\sigma_{\varepsilon}}\right) f_{\Delta X_{n-1, n}}(y) d y \cdot \Phi\left(\frac{d_{P M}-x}{\sigma_{\varepsilon}}\right) f_{X_{n-1}}(x) d x}{\int_{0}^{d_{P M}} \Phi\left(\frac{d_{P M}-x}{\sigma_{\varepsilon}}\right) f_{X_{n-1}}(x) d x} \\
& P\left(F N_{n} \mid F N_{n-1}\right)=P\left\{d_{P M}<X_{n} \leq d_{C M}, Z_{n} \leq d_{P M} \mid d_{P M}<X_{n-1} \leq d_{C M}, Z_{n-1} \leq d_{P M}\right\} \\
& =\frac{\int_{d_{P M}}^{d_{C M}} \int_{d_{P M}-x}^{d_{C M}-x} \Phi\left(\frac{d_{P M}-x-y}{\sigma_{\varepsilon}}\right) f_{\Delta X_{n-1, n}}(y) d y \cdot \Phi\left(\frac{d_{P M}-x}{\sigma_{\varepsilon}}\right) f_{X_{n-1}}(x) d x}{\int_{d_{P M}}^{d_{C M}} \Phi\left(\frac{d_{P M}-x}{\sigma_{\varepsilon}}\right) f_{X_{n-1}}(x) d x} \\
& P\left(X_{n} \geq d_{C M} \mid F N_{n-1}\right)=P\left\{X_{n} \geq d_{C M} \mid d_{P M}<X_{n-1} \leq d_{C M}, Z_{n-1} \leq d_{P M}\right\} \\
& =\frac{\int_{d_{P M}}^{d_{C M}}\left[1-F_{\Delta X_{n-1, n}}\left(d_{C M}-x\right)\right] \cdot \Phi\left(\frac{d_{P M}-x}{\sigma_{\varepsilon}}\right) f_{X_{n-1}}(x) d x}{\int_{d_{P M}}^{d_{C M}} \Phi\left(\frac{d_{P M}-x}{\sigma_{\varepsilon}}\right) f_{X_{n-1}}(x) d x} \\
& P\left(T P_{n} \mid F N_{n-1}\right)=P\left\{d_{P M}<X_{n} \leq d_{C M}, d_{P M}<Z_{n} \leq d_{P M} \mid d_{P M}<X_{n-1} \leq d_{C M}, Z_{n-1} \leq d_{P M}\right\} \\
& =\frac{\int_{d_{P M}}^{d_{C M}} \int_{d_{P M}-x}^{d_{C M}-x} \Phi\left(\frac{d_{P M}-x-y}{\sigma_{\varepsilon}}\right) f_{\Delta X_{n-1, n}}(y) d y \cdot\left[\Phi\left(\frac{d_{C M}-x}{\sigma_{\varepsilon}}\right)-\Phi\left(\frac{d_{P M}-x}{\sigma_{\varepsilon}}\right)\right] f_{X_{n-1}}(x) d x}{\int_{d_{P M}}^{d_{C M}}\left[\Phi\left(\frac{d_{C M}-x}{\sigma_{\varepsilon}}\right)-\Phi\left(\frac{d_{P M}-x}{\sigma_{\varepsilon}}\right)\right] f_{X_{n-1}}(x) d x} \\
& P\left(T P_{n} \mid T N_{n-1}\right)=P\left\{d_{P M}<X_{n} \leq d_{C M}, d_{P M}<Z_{n} \leq d_{P M} \mid X_{n-1} \leq d_{P M}, Z_{n-1} \leq d_{P M}\right\} \\
& =\frac{\int_{0}^{d_{P M}} \int_{d_{P M}-x}^{d_{C M}-x} \Phi\left(\frac{d_{P M}-x-y}{\sigma_{\varepsilon}}\right) f_{\Delta X_{n-1, n}}(y) d y \cdot\left[\Phi\left(\frac{d_{C M}-x}{\sigma_{\varepsilon}}\right)-\Phi\left(\frac{d_{P M}-x}{\sigma_{\varepsilon}}\right)\right] f_{X_{n-1}}(x) d x}{\int_{0}^{d_{P M}}\left[\Phi\left(\frac{d_{C M}-x}{\sigma_{\varepsilon}}\right)-\Phi\left(\frac{d_{P M}-x}{\sigma_{\varepsilon}}\right)\right] f_{X_{n-1}}(x) d x} \\
& P\left(F P_{n} \mid T N_{n-1}\right)=P\left\{X_{n} \leq d_{P M}, d_{P M}<Z_{n} \leq d_{P M} \mid X_{n-1} \leq d_{P M}, Z_{n-1} \leq d_{P M}\right\} \\
& =\frac{\int_{0}^{d_{P M}} \int_{0}^{d_{P M}-x} \Phi\left(\frac{d_{P M}-x-y}{\sigma_{\varepsilon}}\right) f_{\Delta X_{n-1, n}}(y) d y \cdot\left[\Phi\left(\frac{d_{C M}-x}{\sigma_{\varepsilon}}\right)-\Phi\left(\frac{d_{P M}-x}{\sigma_{\varepsilon}}\right)\right] f_{X_{n-1}}(x) d x}{\int_{0}^{d_{P M}}\left[\Phi\left(\frac{d_{C M}-x}{\sigma_{\varepsilon}}\right)-\Phi\left(\frac{d_{P M}-x}{\sigma_{\varepsilon}}\right)\right] f_{X_{n-1}}(x) d x}
\end{aligned}
$$




$$
\begin{gathered}
P\left\{X_{n}<d_{P M} \mid T N_{n-1}\right\}=P\left\{X_{k}<d_{P M} \mid X_{n-1} \leq d_{P M}, Z_{n-1} \leq d_{P M}\right\} \\
=\frac{\int_{0}^{d_{P M}} F_{\Delta X_{n-1, n}}\left(d_{P M}-x\right) \cdot \Phi\left(\frac{d_{P M}-x}{\sigma_{\varepsilon}}\right) f_{X_{n-1}}(x) d x}{\int_{0}^{d_{P M}} \Phi\left(\frac{d_{P M}-x}{\sigma_{\varepsilon}}\right) f_{X_{n-1}}(x) d x} \\
P\left(X_{n} \geq d_{C M} \mid X_{n-1}<d_{P M}\right)=\frac{\int_{0}^{d_{P M}}\left[1-F_{\Delta X_{n-1, n}}\left(d_{C M}-x\right)\right] \cdot f_{X_{n-1}}(x) d x}{F_{X_{n-1}}\left(d_{P M}\right)} \\
P\left(d_{P M} \leq X_{n}<d_{C M} \mid X_{n-1}<d_{P M}\right)=\frac{\int_{0}^{d_{P M}}\left[F_{\Delta X_{n-1, n}}\left(d_{C M}-x\right)-F_{\Delta X_{n-1, n}}\left(d_{P M}-x\right)\right] \cdot f_{X_{n-1}}(x) d x}{F_{X_{n-1}}\left(d_{P M}\right)} \text { (A.10) }
\end{gathered}
$$

